\title{
IMPROVED ASSUMED-STRESS HYBRID SHELL ELEMENT WITH DRILLING DEGREES OF FREEDOM FOR LINEAR STRESS, BUCKLING, AND FREE VIBRATION ANALYSES
}

\author{
Govind Rengarajan* \\ Clemson University, Clemson, SC 29634-0921 \\ Mohammad A. Aminpour ${ }^{\dagger}$ \\ Analytical Services and Materials, Inc., Hampton, VA 23666 \\ Norman F. Knight, Jr. $\ddagger$ \\ Old Dominion University, Norfolk, VA 23529-0247
}

\begin{abstract}
An improved 4-node quadrilateral assumed-stress hybrid shell element with drilling degrees of freedom is presented. The formulation is based on Hellinger-Reissner variational principle and the shape functions are formulated directly for the 4-node element. The element has 12 membrane degrees of freedom and 12 bending degrees of freedom. It has 9 independent stress parameters to describe the membrane stress resultant field and 13 independent stress parameters to describe the moment and transverse shear stress resultant field. The formulation encompasses linear stress, linear buckling, and linear free vibration problems. The element is validated with standard test cases and is shown to be robust. Numerical results are presented for linear stress, buckling, and free vibration analyses.
\end{abstract}

* Graduate Research Assistant, Department of Mechanical Engineering, Currently at Department of Mechanical Engineering, Texas A \& M University, College Station, TX 77843-3123.

$\dagger$ Senior Scientist.

$\ddagger$ Associate Professor, Department of Aerospace Engineering.

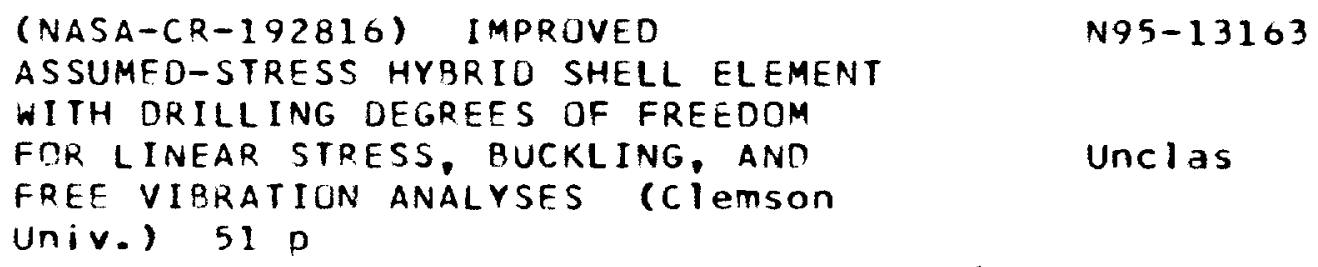




\section{Introduction}

Today, the literature abounds with new shell element formulations and the key issue is the robustness of individual elements. Factors that influence the robustness of elements are spurious zero-energy modes, locking, convergence characteristics, element invariance, and sensitivity to mesh distortion. Considerable progress has been made over the years in addressing some of these factors and in developing suitable assessment procedures and test cases to evaluate and validate the new elements. In the process, several new techniques and new element formulations such as assumedstress, reduced-integration, incompatible modes, and assumed-strain have evolved, that successfully address some of the problems associated with shell elements.

On a geometrical aspect, the shell element formulations that are currently available in literature can be broadly classified into three categories: curved elements based on classical shell theories, degenerated solid shell elements, and flat shell elements. The faceted representation of the shell geometry using flat shell elements is perhaps the simplest of these approaches. These elements combine the membrane and bending behavior of plate elements. While the approximate geometrical representation is immediately evident, particularly in 4-node bilinear elements, the simplicity of the formulation, the convergence characteristics, and full rank has made this approach very attractive. The inclusion of transverse shear effects with the aid of ReissnerMindlin kinematics, and more recently, the inclusion of drilling degrees of freedom $[1,2]$, have significantly improved the performance of flat shell elements.

The assumed-stress hybrid formulations pioneered by Pian and his coworkers [3] is variationally consistent and has led to the development of several powerful element formulations. In general, the mixed/hybrid formulations are computationally more intensive than the displacement-based formulations; however, using symbolic manipulation (e.g., see [4]), and exploiting the banded structure of matrices, the computational cost could be reduced significantly. 
The objective of this paper is to present the formulation of an improved 4-node assumed-stress hybrid shell element with drilling degrees of freedom. The formulation encompasses linear stress, linear buckling, and linear free vibration problems. The element is validated using standard test cases as well as a few other interesting problems for linear stress, buckling, and free vibration analyses. Wherever possible, relevant matrices are generated symbolically, and this has helped save considerable computational time. However, the details of the symbolic manipulation will be dealt with in a subsequent paper.

\section{Drilling Degrees of Freedom}

Drilling degrees of freedom are defined as the rotational degrees of freedom normal to the plane of the element. The drilling rotation $\left(\theta_{z}\right)$ is not represented in the membrane kinematics of the shell. Consequently, the drilling degree of freedom is not included at the element level; however at the global or assembled level, the degrees of freedom associated with the structure may include the drilling degrees of freedom. While assembling the element stiffness matrices to form the global stiffness matrix, if the elements connected to a particular node happen to be coplanar, then the drilling rotation at that node is not resisted. This leads to a singularity in the stiffness matrix [5]. This singularity could clearly be avoided if the drilling degree of freedom is included in the variational statement or in the finite element approximations as will be discussed subsequently. Yet another motivation to include the drilling degree of freedom stems from difficulties in modeling stiffened panels, folded plate structures, etc. The lack of drilling rotation in such instances results in an inadequate representation of the structural response.

Generally, the drilling degrees of freedom are suppressed at the beginning of the analysis since they do not enter the kinematic description of the problem. Zienkiewicz [6] associates a fictitious couple $M_{x}$ with the rotation at the element level to resolve 
the singularity problem. While this does take care of the singularity in the stiffness matrix, it does not utilize the drilling degrees of freedom to improve the finite element approximations. However, there have been numerous attempts in formulating elements with drilling degrees of freedom, and two significant approaches have emerged successful. One approach is to introduce the drilling rotation in the variational statement as an independent variable, while the other approach is to introduce the drilling rotation in the approximations of in-plane displacements.

\section{Drilling Rotation in the Variational Statement}

Reissner [7] presented a mixed variational principle in which the definition of true rotations as the skew-symmetric part of the displacement gradients are relaxed and later enforced in the variational statement as constraints through the introduction of Lagrange multipliers. On imposing the stationary conditions on the functional, the Lagrange multipliers are identified as the skew-symmetric part of the stress tensor. This formulation introduced a rotation field independent of displacements. Hughes and Brezzi [8] modified Reissner's functional by including an additional term that stabilizes the functional in discrete approximations. Ibrahimbegovic et al. $[9,10]$ used the modified formulation of Hughes and Brezzi and developed quadrilateral membrane and flat shell elements. They used independent interpolation fields for rotations and Allman-type [1] shape functions for the in-plane displacements. Iura and Atluri [11] have developed an element where the rotations are interpolated from true nodal rotations evaluated from the skew-symmetric part of the displacement gradient at the nodes.

\section{Drilling Rotations in Finite Element Approximations}

In early attempts, the drilling rotation was introduced in the shape functions using a cubic displacement function (e.g., see Robinson [12]). However, Irons and 
Ahmad [13] pointed out that this method had some difficulties and convergence was not assured. The elements formed in such a manner force the shearing strain to be zero at the nodes and do not pass the patch test. Recently, the drilling rotations have been introduced in the finite element approximations successfully using quadratic displacement functions $[1,2,14-18]$. In this approach, the membrane element is internally assumed to be an 8-node element (four corner nodes and four midside nodes) with 16 degrees of freedom. The stiffness matrix of this 8-node element is then condensed to that of a 4-node element (four corner nodes) with 12 degrees of freedom by associating the displacement degrees of freedom at the midside nodes with the displacement and rotational degrees of freedom at the corner nodes. In effect, the in-plane displacements are dependent on in-plane rotations. MacNeal and Harder [16] have used the above approach to develop a 4 -node membrane element with selectively reduced integration. Yunus et al. [17] have also used this approach to develop assumed-stress hybrid elements. Aminpour $[18,19]$ has developed assumed-stress hybrid shell elements using the Allman-type shape function construction. The formulation of the first element is based on Hellinger-Reissner variational principle. The formulation of the second element is based on modified complementary energy principle and the stress field is expanded in Cartesian coordinates.

\section{Assumed-Stress Hybrid Formulation}

The element developed in this paper is based on Hellinger-Reissner variational principle. The ensuing discussion pertains to a solid elastic continuum $V$, with boundary $S$. Let $S_{\sigma}$ be the section of the boundary where tractions are prescribed; let $S_{u}$ be the section of the boundary where displacements are prescribed. The Hellinger-Reissner functional can be written as

$$
\Pi_{H R}=-\frac{1}{2} \int_{V}\{\sigma\}^{T}[D]\{\sigma\} d V+\int_{V}\{\sigma\}^{T}[\mathcal{L}]\{u\} d V-\int_{S_{\sigma}}\{u\}^{T}\left\{t_{o}\right\} d S,
$$


where $[D]$ is the compliance matrix, $[\mathcal{L}]$ is the linear differential operator on the displacements $\{u\}$ to produce strains, and $\left\{t_{o}\right\}$ is the prescribed tractions on $S_{\sigma}$.

The approximations for stresses and displacements can now be incorporated in the functional. The stress field is described in the interior of the element as

$$
\{\sigma\}=[P]\{\beta\},
$$

and a compatible displacement field is described by

$$
\{u\}=[N]\{q\},
$$

where $[P]$ and $[N]$ are matrices of stress and displacement interpolation functions, respectively, and $\{\beta\}$ and $\{q\}$ are the unknown stress and nodal displacement parameters, respectively. Substituting the stress and displacement approximations [Equations (2) and (3)] in the functional $\Pi_{H R}[$ Equation (1)] results in

$$
\Pi_{H R}=-\frac{1}{2}\{\beta\}^{T}[H]\{\beta\}+\{\beta\}^{T}[T]\{q\}-\{q\}^{T}\{F\},
$$

where

$$
\begin{aligned}
{[H] } & =\int_{V}[P]^{T}[D][P] d V, \\
{[T] } & =\int_{V}[P]^{T}[\mathcal{L}][N] d V, \\
\{F\} & =\int_{S_{\sigma}}[N]^{T}\left\{t_{o}\right\} d S .
\end{aligned}
$$

Now imposing stationary conditions on the functional with respect to the stress parameters $\{\beta\}$ gives

$$
\{\beta\}=[H]^{-1}[T]\{q\} .
$$

On substituting Equation(6) into Equation (4), the functional reduces to

$$
\Pi_{H R}=\frac{1}{2}\{q\}^{T}[K]\{q\}-\{q\}^{T}\{F\},
$$

where $[K]$, the elemental stiffness matrix, is given by

$$
[K]=[T]^{T}[H]^{-1}[T] .
$$


Imposing the stationary conditions on the functional with respect to nodal displacement unknowns $\{q\}$, results in a system of linear algebraic equations of the form

$$
[K]\{q\}=\{F\} .
$$

\section{Finite Element Approximations}

\section{Displacement Field}

In the present paper, the Allman-type shape functions are used. However, the formulation is direct rather than forming the stiffness matrix of an 8-node element and then condensing it to a 4-node element stiffness matrix. All four edges of the quadrilateral may be treated as 2-D beam elements with shear deformation, and the general interpolation function for any typical edge can be expressed as in reference [18] by

$$
\begin{aligned}
u^{o} & =\frac{1}{2}(1-\xi) u_{i}+\frac{1}{2}(1+\xi) u_{j}+\frac{\Delta y_{i}}{8}\left(1-\xi^{2}\right)\left(\theta_{z j}-\theta_{z i}\right) \\
v^{0} & =\frac{1}{2}(1-\xi) v_{i}+\frac{1}{2}(1+\xi) v_{j}-\frac{\Delta x_{i}}{8}\left(1-\xi^{2}\right)\left(\theta_{z j}-\theta_{z i}\right)
\end{aligned}
$$

and

$$
\begin{aligned}
& \Delta x_{i}=x_{j}-x_{i}, \\
& \Delta y_{i}=y_{j}-y_{i},
\end{aligned}
$$

where $i=1,2,3,4$ and $j=2,3,4,1$ in that order. As mentioned earlier, the drilling rotations are not interpolated independently. The out-of-plane displacement and outof-plane rotations are given by

$$
\begin{aligned}
w^{o}= & \frac{1}{2}(1-\xi) w_{i}+\frac{1}{2}(1+\xi) w_{j}-\frac{\Delta y_{i}}{8}\left(1-\xi^{2}\right)\left(\theta_{x j}-\theta_{x i}\right), \\
& +\frac{\Delta x_{i}}{8}\left(1-\xi^{2}\right)\left(\theta_{y j}-\theta_{y i}\right) \\
\theta_{x}= & \frac{1}{2}(1-\xi) \theta_{x i}+\frac{1}{2}(1+\xi) \theta_{x j}, \\
\theta_{y}= & \frac{1}{2}(1-\xi) \theta_{y i}+\frac{1}{2}(1+\xi) \theta_{y j} .
\end{aligned}
$$


Note that all three displacement degrees of freedom and two in-plane rotations are approximated by quadratic and linear functions, respectively. This difference in the order of approximating polynomials between displacements and rotations automatically alleviates shear locking. Equations (10) and (11) can be extended directly to all four sides of the quadrilateral element to obtain the interpolation functions for the element. The generalized displacements represented in terms of interpolation functions are

$$
\begin{aligned}
& u^{o}(\xi, \eta)=\sum_{i=1}^{4} N_{i} u_{i}+\sum_{i=1}^{4} \frac{\Delta y_{i}}{8} N_{i}^{*}\left(\theta_{z j}-\theta_{z i}\right) \\
& v^{o}(\xi, \eta)=\sum_{i=1}^{4} N_{i} v_{i}-\sum_{i=1}^{4} \frac{\Delta x_{i}}{8} N_{i}^{*}\left(\theta_{z j}-\theta_{z i}\right) \\
& w^{o}(\xi, \eta)=\sum_{i=1}^{4} N_{i} w_{i}-\sum_{i=1}^{4} \frac{\Delta y_{i}}{8} N_{i}^{*}\left(\theta_{x j}-\theta_{x i}\right)+\sum_{i=1}^{4} \frac{\Delta x_{i}}{8} N_{i}^{*}\left(\theta_{y j}-\theta_{y i}\right), \\
& \theta_{x}(\xi, \eta)=\sum_{i=1}^{4} N_{i} \theta_{x i}, \\
& \theta_{y}(\xi, \eta)=\sum_{i=1}^{4} N_{i} \theta_{y i}
\end{aligned}
$$

where

$$
\begin{gathered}
N_{i}=\frac{1}{4}\left(1+\xi_{i} \xi\right)\left(1+\eta_{i} \eta\right) ; i=1,2,3,4 \\
N_{i}^{*}=\left\{\begin{array}{lc}
\frac{1}{2}\left(1-\xi^{2}\right)\left(1+\eta_{i} \eta\right) & ; i=1,3 \\
\frac{1}{2}\left(1-\eta^{2}\right)\left(1+\xi_{i} \xi\right) & ; i=2,4
\end{array}\right.
\end{gathered}
$$

and

$$
j=\left\{\begin{array}{cl}
i+1 & ; i=1,2,3 \\
1 \quad ; \quad i=4
\end{array}\right.
$$

In the above set of equations $\xi$ and $\eta$ are the element natural coordinates and $\xi_{i}$ and $\eta_{i}$ are the values of $\xi$ and $\eta$ at node $i$. Note that the drilling degrees of freedom $\left(\theta_{x i}\right)$ are not true nodal rotations but are referred to as "rotational connectors" [1] and enter the finite element approximations as differences in rotational connectors.

As opposed to conventional 4-node shell elements which have 20 degrees of freedom, elements with drilling degrees of freedom have 24 degrees of freedom. The 
membrane part of the element has 12 degrees of freedom. Associated with these 12 degrees of freedom are 12 independent deformation modes. Three of these modes are rigid-body modes and three other modes represent constant strain states. Of the remaining six modes, five represent higher-order strain states and the last mode is a spurious zero-energy mode. The spurious zero-energy mode associated with this formulation is caused by the drilling rotations or rather the rotational connectors. Note from Equations (10),(11) and (12) that the differences in rotations, and not the drilling rotations themselves, enter the interpolation functions [see Equation (12)]. One of the four rotational differences is dependent on the other three rotational differences, and hence, there are only three independent rotational degrees of freedom. This produces a rank deficiency in the stiffness matrix and leads to a spurious zeroenergy mode. This spurious mode is associated with a state of zero nodal in-plane displacements and equal nodal in-plane rotations.

However, this mode can be suppressed easily. MacNeal and Harder [16] eliminate this mode by adding an energy penalty to the stiffness matrix. The energy penalty is defined in terms of the weighted average of the differences between corner rotations and the rotation at the center of the element. The rotation at the center of the element is obtained by evaluating the skew-symmetric part of the displacement gradient at the center of the element. A simpler way to eliminate this spurious mode, but which calls for the awareness of any user of the finite element, is to prescribe the value of the in-plane rotation at one node in the entire domain being analyzed. This will remove the rank deficiency in the membrane part of the stiffness matrix.

The bending part of the element also has 12 degrees of freedom. Similar to the membrane part, 12 independent deformation modes are associated with these 12 degrees of freedom. Three modes represent rigid-body modes, five modes represent constant strain states, the remaining four modes represent higher-order strain states. A spurious mode similar to the one in the membrane part does not occur here, though 
the interpolation function for out-of-plane displacement $w$ [Equation (11)] contains out-of-plane (bending) rotational differences. In the membrane part, the rotational degrees of freedom, as rotational differences, enter the formulation through the interpolation functions for in-plane displacements and not independently. However, in the bending part, the out-of-plane rotations enter the formulation both independently $\left[2^{\text {nd }}\right.$ and $3^{\text {rd }}$ of Equation (11)] and through the interpolation function for $w$. Thus, the bending part of the stiffness matrix is not rank deficient.

\section{Assumed-Stress Field}

In general, the selection of stress field approximation is governed by a few conditions and guidelines [20] such as

- the satisfaction of equilibrium equations,

- interelement traction reciprocity,

- invariance and completeness of polynomial expansion, and

- spurious zero-energy modes.

In earlier hybrid models based on the principle of minimum complementary energy [21], the assumed-stress field satisfied equilibrium equations exactly, and consequently the stress field was expanded in Cartesian coordinates. However, in the hybrid formulations such as the present one, the assumed-stress field can be expanded in element natural-coordinate basis and the equilibrium equations need not be satisfied a priori. In any case, the equilibrium equations are satisfied in a variational sense. The invariance of the assumed-stress field could be achieved by having a complete polynomial expansion [20]. However, the expansion of assumed-stress field in element natural-coordinate basis provides a way to achieve the necessary invariance property to the approximations.

Finally, the chosen stress field should produce no spurious zero-energy modes. Let $N a$ be the number of independent stress parameters in the stress field approximations; 
let $N_{d}$ be the number of deformation modes associated with the element; let $N_{r}$ be the number of rigid-body modes associated with the element. In order to suppress each independent deformation mode, it is necessary that a corresponding stress term exists such that the deformation mode does not produce zero strain energy. As such, each independent stress parameter should suppress one non-trivial independent deformation mode $[20,22]$. That is,

$$
N_{s} \geq\left(N_{d}-N_{r}\right)
$$

While this statement sets a lower bound on $N_{0}$, a definitive statement regarding the upper bound on $N$, cannot be made here. In this context, it is useful to define a ratio ' $\alpha$ ' given by

$$
\alpha=\frac{N_{s}}{N_{d}-N_{r}}
$$

Based on Equation (16), it is clear that $\alpha \geq 1$. At this stage, a stress field that is described by a complete polynomial expansion in element natural-coordinate basis and that does not satisfy equilibrium equations a priori might appear suitable. However, it has been observed that a very high value of $\alpha$ produces a very stiff element [23]. Fraejis de Veubeke's limitation principle [25] states that when a complete independent stress field that does not satisfy equilibrium equations a priori is assumed, the hybrid element would reduce to a fully integrated displacement-based element. One way to overcome this is to impose additional constraints by enforcing equilibrium in the variational statement using Lagrange multipliers [3]. The lower bound on $\alpha$ in mixed/hybrid formulations is stated mathematically by the inf - sup condition, popularly known as the LBB condition [24].

In the present approach the assumed-stress field is not complete, and the equilibrium equations are satisfied exactly for regular (rectangular) element shapes. The stress field for the membrane part is assumed to be

$$
N_{\xi}=\beta_{1}+\beta_{4} \xi+\beta_{6} \eta+\beta_{8} \eta^{2}
$$




$$
\begin{aligned}
& N_{\eta}=\beta_{2}+\beta_{5} \xi+\beta_{7} \eta+\beta_{9} \xi^{2}, \\
& N_{\xi \eta}=\beta_{3}-\beta_{4} \eta-\beta_{7} \xi .
\end{aligned}
$$

The stress field is expanded in natural coordinates to make the element less sensitive to mesh distortion. Also note that the stress field is invariant with respect to reference natural coordinates. The stress resultants expressed in natural coordinates are transformed to Cartesian coordinates by the contravariant tensor transformation laws [3]. The transformation rule for the stress field is

$$
\sigma_{(c)}^{\alpha \beta}=J_{\gamma}^{\alpha}(\xi, \eta) J_{\delta}^{\beta}(\xi, \eta) \sigma_{(n)}^{\gamma \delta}(\xi, \eta)
$$

where $J_{m}^{n}$ are terms from the Jacobian matrix, given by

$$
J_{m}^{n}=\frac{\partial x_{n}}{\partial \xi_{m}}
$$

and $\sigma_{(c)}^{\alpha \beta}$ and $\sigma_{(n)}^{\gamma \delta}$ represent the stress state in Cartesian coordinates and natural coordinates, respectively. For a distorted mesh, $J_{m}^{n}$ will be linear in $\xi$ and/or $\eta$ and hence the transformation involving $J_{\gamma}^{\alpha} J_{\delta}^{\beta}$ will have higher-order terms in it. Moreover, the constant stress terms will no longer be preserved which causes the element to fail the constant strain patch test. In order to preserve the order of stress field chosen, including the constant terms, $J_{m}^{n}$ 's are evaluated at the origin of the naturalcoordinate system of the element. Now the transformation rule is

$$
\sigma_{(\boldsymbol{c})}^{\alpha \beta}=J_{\gamma}^{\alpha}(0,0) J_{\delta}^{\beta}(0,0) \sigma_{(n)}^{\gamma \delta}(\xi, \eta)
$$

This transformation is used in evaluating both the $[H]$ and $[T]$ matrices [Equation (5)] needed for the linear stiffness matrix $[K]$ [Equation (8)].

The stress field described in Equation (18) will not satisfy equilibrium equations a priori for any general, irregular shape. However, this field satisfies equilibrium equations a priori for regular (rectangular) element shapes. In any case, as discussed earlier, the stress field will satisfy the equilibrium equations in a variational sense. 
The bending part of the element also has 12 degrees of freedom and hence 12 independent deformation modes. Three modes are rigid-body modes. Similar to the membrane part, 9 independent stress parameters are required to suppress all the other deformation modes. Though 9 independent stress parameters are enough to suppress all the deformation modes, the following 13-parameter stress field for the bending part is less sensitive to mesh distortion,

$$
\begin{aligned}
M_{\xi} & =\bar{\beta}_{1}+\bar{\beta}_{4} \xi+\bar{\beta}_{6} \eta+\bar{\beta}_{8} \eta^{2}, \\
M_{\eta} & =\bar{\beta}_{2}+\bar{\beta}_{5} \xi+\bar{\beta}_{7} \eta+\bar{\beta}_{9} \xi^{2}, \\
M_{\xi \eta} & =\bar{\beta}_{3}+\bar{\beta}_{10} \xi+\bar{\beta}_{11} \eta+\frac{1}{2} \bar{\beta}_{12} \xi^{2}+\frac{1}{2} \bar{\beta}_{13} \eta^{2} .
\end{aligned}
$$

This 13-parameter stress field is a little different from the one used in reference [18]. Numerical experimentations indicate that this field is somewhat more accurate than the one used in reference [18].

The transverse shear stress resultants are obtained by relating the transverse shear stress resultants to bending stress resultants, as

$$
\begin{aligned}
& Q_{\xi}=\bar{\beta}_{4}+\bar{\beta}_{11}+\bar{\beta}_{13} \eta \\
& Q_{\eta}=\bar{\beta}_{7}+\bar{\beta}_{10}+\bar{\beta}_{12} \xi .
\end{aligned}
$$

Note that the transverse shear stress resultant field is coupled to bending stress resultant field and the equilibrium equations are satisfied a priori for regular (rectangular) shapes. The stress fields [Equations (21),(22)] are transformed to Cartesian coordinate system using the same contravariant tensor transformation laws used for the membrane part [Equation (20)]. Also note that $\alpha=1$ [Equation (17)] for membrane, $\alpha=1.44$ for bending, and $\alpha=1.22$ for membrane and bending combined. 


\section{Geometric Stiffness Matrix}

The geometric stiffness or initial stress matrix is derived by using the full nonlinear Green-Lagrange strain tensor

$$
\epsilon_{i j}=\frac{1}{2}\left(\frac{\partial u_{i}}{\partial x_{j}}+\frac{\partial u_{j}}{\partial x_{i}}+\frac{\partial u_{k}}{\partial x_{i}} \frac{\partial u_{k}}{\partial x_{j}}\right) .
$$

Using Reissner-Mindlin kinematic assumptions and retaining the nonlinear terms only for the membrane strains, the following relations result. The strains are written as

$$
\{\epsilon\}=\left\{\epsilon^{\circ}\right\}+z\{\kappa\}
$$

where $\left\{\epsilon^{o}\right\}$ are the membrane strains, $\{\kappa\}$ are the bending strains, and $z$ is the coordinate in the direction through the thickness of the plate or shell. The membrane strains are written as

$$
\left\{\epsilon^{o}\right\}=\left\{\epsilon_{L}^{o}\right\}+\left\{\epsilon_{N L}^{o}\right\}
$$

where the linear part is given by

$$
\left\{\epsilon_{L}^{o}\right\}=\left\{\begin{array}{c}
\epsilon_{x}^{o} \\
\epsilon_{y}^{o} \\
\epsilon_{x y}^{o}
\end{array}\right\}=\left\{\begin{array}{c}
\partial u^{o} / \partial x \\
\partial v^{o} / \partial y \\
\partial u^{o} / \partial y+\partial v^{o} / \partial x
\end{array}\right\}
$$

and the nonlinear part is given by

$$
\left\{\epsilon_{N L}^{o}\right\}=\frac{1}{2}\left\{\begin{array}{c}
\left(\frac{\partial u^{\circ}}{\partial x}\right)^{2}+\left(\frac{\partial v^{\circ}}{\partial x}\right)^{2}+\left(\frac{\partial w^{\circ}}{\partial x}\right)^{2} \\
\left(\frac{\partial u^{\circ}}{\partial y}\right)^{2}+\left(\frac{\partial v^{\circ}}{\partial y}\right)^{2}+\left(\frac{\partial w^{\circ}}{\partial y}\right)^{2} \\
2\left(\frac{\partial u^{\circ}}{\partial x} \frac{\partial u^{\circ}}{\partial y}+\frac{\partial v^{y}}{\partial x} \frac{\partial v^{\circ}}{\partial y}+\frac{\partial w^{\circ}}{\partial x} \frac{\partial w^{\circ}}{\partial y}\right)
\end{array}\right\} .
$$

The bending strains are computed using the changes in curvature by

$$
\{\kappa\}=\left\{\begin{array}{c}
\kappa_{x} \\
\kappa_{y} \\
\kappa_{x y}
\end{array}\right\}=\left\{\begin{array}{c}
\partial \theta_{y} / \partial x \\
-\partial \theta_{x} / \partial y \\
\partial \theta_{y} / \partial y-\partial \theta_{x} / \partial x
\end{array}\right\} .
$$

The transverse shear strains are given by

$$
\left\{\gamma^{o}\right\}=\left\{\begin{array}{c}
\gamma_{x z}^{o} \\
\gamma_{y x}^{o}
\end{array}\right\}=\left\{\begin{array}{r}
\theta_{y}+\partial w^{o} / \partial x \\
-\theta_{x}+\partial w^{o} / \partial y
\end{array}\right\} .
$$


The generalized Hellinger-Reissner functional including the nonlinear strains can be written as

$$
\begin{aligned}
\Pi_{H R}= & -\frac{1}{2} \int_{V}\{\sigma\}^{T}[D]\{\sigma\} d V+\int_{V}\{\sigma\}^{T}[\mathcal{L}]\{u\} d V \\
& +\int_{V}\left\{\sigma_{0}\right\}^{T}\left\{\epsilon_{N L}^{o}\right\} d V-\int_{S_{\sigma}}\{u\}^{T}\left\{t_{o}\right\} d S
\end{aligned}
$$

where $\left\{\sigma_{0}\right\}$ is the prescribed pre-buckling stress state. Upon substitution of the displacement and stress approximations, Equation (30) reduces to

$$
\Pi_{H R}=-\frac{1}{2}\{\beta\}^{T}[H]\{\beta\}+\{\beta\}^{T}[T]\{q\}-\{q\}^{T}\{F\}+\frac{1}{2}\{q\}^{T}\left[K_{\sigma}\right]\{q\},
$$

where the element geometric stiffness matrix $\left[K_{\sigma}\right]$ is given by

$$
\left[K_{\sigma}\right]=\int_{A}[N]^{T}[G]^{T}[\mathcal{S}][G][N] d A
$$

and

$$
[G]=\left[\begin{array}{cccccc}
\partial_{x} & 0 & 0 & 0 & 0 & 0 \\
\partial_{y} & 0 & 0 & 0 & 0 & 0 \\
0 & \partial_{x} & 0 & 0 & 0 & 0 \\
0 & \partial_{y} & 0 & 0 & 0 & 0 \\
0 & 0 & \partial_{x} & 0 & 0 & 0 \\
0 & 0 & \partial_{y} & 0 & 0 & 0
\end{array}\right]
$$

where $\partial_{x}=\partial / \partial x$ and $\partial_{y}=\partial / \partial y$. The matrix $[\mathcal{S}]$, which corresponds to the prebuckling stress state, consists of membrane stress resultants which are either prescribed or evaluated from a linear static stress analysis. It is given by

$$
[\mathcal{S}]=\left[\begin{array}{lll}
\mathbf{S} & 0 & 0 \\
0 & \mathbf{S} & 0 \\
0 & 0 & \mathbf{S}
\end{array}\right]
$$

where

$$
\mathrm{S}=\left[\begin{array}{cc}
N_{x}^{o} & N_{x y}^{o} \\
N_{x y}^{o} & N_{y}^{o}
\end{array}\right]
$$

\section{Consistent Mass Matrix}

In linear free vibration analysis, it is assumed that the system is undamped and there are no external forces. The Hellinger-Reissner functional [Equation (1)] with the 
kinetic energy contribution can be written as

$$
\begin{aligned}
\Pi_{H R}= & \int_{t_{1}}^{t_{2}}\left(-\frac{1}{2} \int_{V}\{\sigma\}^{T}[D]\{\sigma\} d V+\int_{V}\{\sigma\}^{T}[\mathcal{L}]\{u\} d V\right. \\
& \left.-\frac{1}{2} \int_{V}\{\dot{u}\}^{T}[\rho]\{\dot{u}\} d V\right) d t
\end{aligned}
$$

where dots (.) over $u$ indicate time derivatives. Upon substitution of stress field and displacement field approximations, Equation (34) reduces to

$$
\Pi_{H R}=\int_{t_{1}}^{t_{2}}\left(-\frac{1}{2}\{\beta\}^{T}[H]\{\beta\}+\{\beta\}^{T}[T]\{q\}-\frac{1}{2}\{\dot{q}\}^{T}\left[M_{c}\right]\{\dot{q}\}\right) d t,
$$

where the consistent mass matrix $\left[M_{c}\right]$, representing the inertia of the system, is given by

$$
\left[M_{c}\right]=\int_{A}[N]^{T}[\bar{\rho}][N] d A
$$

and

$$
[\bar{\rho}]_{5 \times 5}=\left[\begin{array}{rrrrr}
m_{o} & 0 & 0 & 0 & m_{1} \\
0 & m_{o} & 0 & -m_{1} & 0 \\
0 & 0 & m_{o} & 0 & 0 \\
0 & -m_{1} & 0 & m_{2} & 0 \\
m_{1} & 0 & 0 & 0 & m_{2}
\end{array}\right]
$$

where

$$
\begin{aligned}
& m_{0}=\int_{-\frac{h}{2}}^{\frac{h}{2}} \rho d z, \\
& m_{1}=\int_{-\frac{h}{2}}^{\frac{h}{2}} \rho z d z \\
& m_{2}=\int_{-\frac{h}{2}}^{\frac{h}{2}} \rho z^{2} d z
\end{aligned}
$$

and $\rho$ is the mass density.

\section{Numerical Results}

The element described herein was developed and implemented within the framework of NASA Langley Research Center's computational structural mechanics testbed COMET [26] using the generic element processor template [27]. The element will be referred to herein as A4S1. 
The proper selection of displacement and stress fields has automatically eliminated spurious zero-energy modes and locking in the present element and has made the element invariant with respect to reference coordinates. However, the element still needs to be validated with regard to its convergence and its sensitivity to mesh distortion. MacNeal and Harder [28] have collected and proposed a standard set of problems to test and validate a new element. The element's performance is observed by analyzing these problems and a few other classical problems. The results are compared with known theoretical solutions and other numerical solutions. Other 4-node quadrilateral shell elements used for comparison are briefly described in Appendix A. Consistent units have been used throughout the analyses.

\section{Patch Test}

This test was originally proposed by Irons [13] to establish the convergence of an element. The patch test as shown in Figure 1 involves a specific mesh of distorted elements with specified applied displacements that result in a constant state of either a membrane or bending stress field. It is now widely recognized that an element should pass the patch test, failing which, its convergence will not be assured. A4S1 passes both the membrane and bending patch tests. The recovered strains and stresses are exact.

\section{MacNeal-Harder Straight Cantilever Beam}

This test case was proposed by MacNeal and Harder [28]. The capability of the element to handle constant and linearly varying strains and curvatures is tested by applying appropriate unit loads at the free end. The beam is modeled by a $6 \times 1$ mesh. It is discretized using rectangular-, trapezoidal- and parallelogram-shaped elements. The element's sensitivity to mesh distortion is studied and compared with other elements. The finite element discretizations and the exact solutions [28] are 
shown in Figure 2. The theoretical result for twist (load D) is 0.03406 as reported in Timoshenko and Goodier [29]. However, reference [28] reports the solution to be 0.03028 . Analysis with successively refined meshes converged to 0.03385 which is much closer to Timoshenko and Goodier solution [29], and hence this solution is used here for normalization. The normalized results given in Table I indicate that the elements predict good results for rectangular-shaped elements and the results deteriorate for the two distorted mesh cases. However, A4S1 performs very well and can handle distorted mesh configurations effectively.

- Remark 1: Attention is drawn to the case of in-plane shearing load (load B), particularly in distorted mesh configurations. 4_ANS, 4_HYB, and QUAD4 perform badly. The advantage of drilling degrees of freedom is evident from the superior performance of the present element, the performance of Q4S, and a relatively modest improvement shown by 4 STTG.

- Remark 2: A4S1 has shown improvement over AQR8 and AQD4 for the twist (load D), and this is attributed to the modified bending stress field.

- Remark 3: The rotational degrees of freedom by virtue of their presence in the element displacement shape functions, enter into the calculations of workequivalent nodal loads. Hence, the in-plane and out-of-plane loads at the beam tip correspond to both statically equivalent loads and the work-equivalent loads including moments corresponding to the rotational degrees of freedom.

\section{Twisted Cantilever Beam}

This test case involves a straight cantilever beam twisted $90^{\circ}$ over its length [28]. The twisted beam shown in Figure 3 is modeled by 12 elements along the length and 2 elements across the width. Each element has a $7.5^{\circ}$ warp and the effect of this warp on the element's performance is studied. The results tabulated in Table II indicate that A4S1 performs very well, but does not show improvement over AQR8 and AQD4. While 4_STG and QUAD4 perform well, 4_ANS and 4_HYB do not perform very well. 


\section{Curved Cantilever Beam}

The curved cantilever beam formed by a $90^{\circ}$ circular arc is shown in Figure 4 . The loading consists of in-plane shear and out-of-plane shear at the tip [28]. The results tabulated in Table III indicate that the new element performs very well. Again A4S1 has shown a modest improvement over AQR8 and AQD4 for the out-of-plane shear (load C) and this is again attributed to the improved bending stress field.

\section{Scordelis-Lo Roof}

The Scordelis-Lo roof $[30]$ is a singly curved shell structure (see Figure 5). The shell has a gravity load or a uniform dead load (UDL), and the parameter of interest is the vertical displacement at the mid-point of the free edge (point $A$ in Figure 5). Though the theoretical value used in reference [30] is 0.3086 , the value used by MacNeal and Harder [28] is 0.3024 . This latter value is used for normalization here. Due to the symmetry of the structure and loading, only one quadrant of the shell is modeled. The convergence behavior is studied for meshes from $2 \times 2$ to $10 \times 10$ and is tabulated in Table IV. The A4S1 element shows a rapid and monotonic convergence.

\section{Morley's Spherical Shell}

The spherical shell is doubly curved (see Figure 6) and the equator of the shell is chosen to be a free edge. Hence, the problem reduces to a hemisphere with four point loads alternating in sign at $90^{\circ}$ intervals along the equator. An $18^{\circ}$ hole has been introduced at the top of the hemisphere to avoid having to model the pole [28]. The theoretical solution for displacement at the point of load and in the direction of the load is reported as 0.094 in reference [28] and it is used here for normalization. Only a quadrant of the shell is modeled. Convergence is studied for meshes from $2 \times 2$ to $12 \times 12$ and the results are tabulated in Table V. While 4_ANS and QUAD4 seem 
to show a very rapid convergence, A4S1, AQR8, AQD4, and 4_STG exhibit a slow convergence. Note that even for a $12 \times 12$ mesh, A4S1 shows an $8.4 \%$ error.

- Remark 4: The present problem involves significant contribution from both membrane and bending strains to the radial displacement at the points of loading. The reason attributed for the slow convergence of the elements with drilling rotations [16] is that the stiffness of the shell is increased by membranebending coupling arising out of the faceted geometric approximation of the shell. This coupling is actually the coupling between drilling rotations and bending rotations due to the changes in slopes at element interfaces (because of faceted representation).

- Remark 5: Furthermore, the membrane-bending coupling may be amplified by the fact that the drilling rotations are not true rotations, but are rotational connectors. An independent drilling rotation entering the formulation either through the variational statement or through the kinematics may increase the flexibility of the discretized shell.

\section{Isotropic Square Plate}

The classical plate bending problem of a clamped, isotropic square plate subjected to a concentrated load at its center is analyzed. The convergence behavior, the effect of mesh distortion, and the effect of varying the thickness of the plate are studied. The geometry of the plate and its properties are shown in Figure 7. The maximum deflection at the center of the plate, as calculated in reference [31] using the Ritz approximation, is given by

$$
W_{\text {max. }}^{\text {anal. }}=0.0056 \frac{P a^{2}}{D}
$$

where $D$ is the flexural rigidity of the plate, $P$ is the applied concentrated load for the full plate, and $a$ is the side length of the plate. The central deflections obtained from the finite element analyses are normalized with respect to the value calculated from Equation 39.

The convergence behavior shown in Figure 7 indicates that $\mathrm{A} 4 \mathrm{~S} 1$ converges rapidly and monotonically. Next, the analysis is carried out using a distorted $7 \times 7$ mesh. The center of the quarter plate is fixed and the nodes immediately adjacent to 
the central node are rotated from 0 to 45 degrees. The boundary nodes are also fixed. From Figure 8, the mesh distortion is shown to have the least effect on A4S1. Again A4S1 offers a modest improvement over AQR8 and AQD4. As mentioned earlier, the consistent interpolation order between the transverse displacement and out-of-plane rotations eliminates shear locking in $\mathrm{A} 4 \mathrm{~S} 1$. To confirm this with numerical validation, the effect of decreasing the thickness of the plate on the element's performance is studied. The length-to-thickness ratio (defined as $a / h$ ) is varied from 10 to 10,000 . None of the elements lock at high length-to-thickness ratio. Representative results for two elements are shown in Figure 9.

\section{Pear-Shaped Cylinder}

This is an extremely interesting problem because of its continually varying geometry. The cross-section of the cylinder is shown in Figure 10, which supposedly is a representation of an early space shuttle fuselage configuration. The shell is isotropic with a uniform thickness of 0.01 inches. Only one quarter of the cylinder is modeled due to the symmetry of the structure. Symmetry boundary conditions are imposed on three edges and a uniform end shortening is applied to the simply supported edge. The analysis is carried out using three different meshes: $4 \times 23$ (92 nodes, 66 elements), $5 \times 34$ (170 nodes, 132 elements), and $7 \times 51$ (357 nodes, 300 elements), where $n \times m$ refers to $n$ nodes along the length of the cylinder and $m$ nodes along the half-cylinder circumference. However, the solution reported here is for the $7 \times 51$ mesh, where the solutions converge.

One of the earliest available numerical analyses on this problem was performed by Hartung and Ball [32]. The variation of radial displacements and the axial stress resultants with $\theta$ measured counter-clockwise from the top, are shown in Figures 11 and 12, respectively. Results of 4_ANS and A4S1 are very close. Further comments on the accuracy of the solution cannot be made here due to the lack of linear static 
analysis data on this problem. However, this problem has been an attractive target for nonlinear analyses and elastic shell collapse studies, due to its complex nonlinear collapse behavior. Herein a linear elastic buckling analysis is carried out on the cylinder. The buckling loads for two modes are presented in Table VI and the buckling mode shapes are shown in Figure 13. Since there are no analytical solutions available for comparison, the results are normalized with respect to converged solutions of a 9-node assumed natural strain element (9_ANS) $[26,33]$. The present element and 4_STG which contain the drilling degree of freedom, predict lower buckling loads compared to 4_ANS.

\section{Rectangular Plate}

This problem is analyzed to validate the element's capability to handle linear buckling and free vibration problems. This is a classical problem for which theoretical solutions exist. The rectangular plate [see Figure 14] is isotropic and simply supported on all sides. The plate is subjected to a uniform uniaxial compression and a biaxial compression [see Figure 14(a) and 14(b), respectively]. Only a quarter of the plate is discretized, with a $5 \times 8$ mesh [shaded region in Figure 14(a) and 14(b)]. The linear buckling analysis is performed with the pre-buckling stress state consisting of stresses computed from a linear static analysis. The results are tabulated in Table VII and VIII, where $m$ and $n$ refer to the number of half waves in $X$ and $Y$ directions, respectively. The results are normalized with respect to the theoretical solutions reported in reference [34]. A4S1 predicts quite accurate buckling loads and again, A4S1 and 4_STG predict lower buckling loads than 4_ANS. The plate is also subjected to an uniform shear and the full plate is discretized with a $5 \times 8$ mesh [see Figure 14(c)]. The results are shown in Table IX. A4S1 predicts a lower and more accurate buckling load than 4_ANS. 
A free vibration analysis is carried out on the plate in its unstressed state. The results are tabulated in Table $\mathrm{X}$, where $m$ and $n$ refer to the number of half waves in $X$ and $Y$ directions, respectively. The results are normalized with respect to the theoretical solutions reported in reference [34]. A4S1 gives quite accurate natural frequencies, but 4_HYB seems to have performed marginally better.

\section{Axially Compressed Cylinder}

A cylindrical shell with simply supported edges is subjected to an uniform axial compression (see Figure 15). The prestress state is computed from a linear stress analysis of the axially compressed cylinder. Note that this prestress state is not exactly constant due to the simply supported boundaries, which prevents the shell from uniformly expanding in circumferential direction. Another interesting feature of this problem is the closely packed eigenvalues and the corresponding considerably different mode shapes. Modeling the entire cylinder is computationally intensive and hence only a 15-degree sector and one tenth of the length of the cylinder, sufficient to capture the lowest mode, is modeled. Symmetric boundary conditions are employed on all edges except the loaded edge which is simply supported. A convergence study

is done for meshes $\mathrm{N} \times \mathrm{N}$, where $\mathrm{N}$ (number of nodes per side) ranges from 3 to 9 (see Figure 15). A4S1 and 4_STG show a better convergence than 4_ANS. The first two mode shapes of this discretization are shown in Figure 16.

\section{Cylindrical Shell}

An octant of a cylindrical shell (see Figure 17) is analyzed for the free vibrational characteristics of the cylindrical shell. To capture the correct modes, symmetric and anti-symmetric boundary conditions are defined for the edge $\mathrm{BC}$. Edge $\mathrm{AB}$ is simply supported and Edges $A D$ and $D C$ are in the symmetric planes. The results are compared with the theoretical solutions reported in reference [35] and are tabulated 
in Table XI. The mesh refers to number of nodes in the circumferential direction by number of nodes in the axial direction. A4S1 and $4 \mathrm{HYB}$ converge to the theoretical solution. The vibration mode shapes are shown in Figure 17.

\section{Conclusions}

Numerical results indicate that the element developed in this paper is devoid of the usual deficiencies of 4-node shell elements. Though the element has one spurious zero-energy mode, this mode can be suppressed easily by prescribing the value of in-plane normal rotation at one node in the entire finite element model. It has been shown that the element is nearly insensitive to mesh distortion, does not lock, and has desirable convergence and invariance properties. The element has also performed very well for buckling and free vibration analyses.

\section{Acknowledgment}

The research reported herein was sponsored by NASA Langley Research Center and Dr. Alexander Tessler was the technical monitor. The first and third authors were sponsored by NASA Grant NAG-1-1374, and the second author was sponsored by NASA Contract NAS1-19317. 


\section{References}

[1] Allman, D.J., "A Compatible Triangular Element Including Vertex Rotations for Plane Elasticity Analysis", Computers and Structures, Vol. 19, 1984, pp. 1-8.

[2] Bergan, P.G., and Felippa, C.A., "A Triangular Element with Rotational Degrees of Freedom", Computer Methods in Applied Mechanics and Engineering, Vol. 50, 1985, pp. 25-69.

[3] Pian, T.H.H., "Evolution of Assumed Stress Hybrid Finite Element", in Accuracy, Reliability and Training in FEM Technology, Proceedings of the Fourth World Congress and Exhibition on Finite Element Methods, Edited by John Robinson, Interlaken, Switzerland, 1984, pp. 602-619.

[4] Tan, H.Q., Chang, T.Y.P., and Zheng, D., "On Symbolic Manipulation and Code Generation of a Hybrid Three-dimensional Solid Element", Engineering with Computers, Vol. 7, 1991, pp. 47-59.

[5] Cook, R.D., Malkus, D.S., and Plesha, M.E., Concepts and Applications of Finite Element Analysis, Third Edition, John Wiley and Sons Inc., New York, 1989.

[6] Zienkiewicz, O.C., The Finite Element Method, Fourth Edition, Vol. 1, McGrawHill, New York, 1988.

[7] Reissner, E., "A Note on Variational Principles in Elasticity", International Journal of Solids and Structures, Vol. 1, 1965, pp. 93-95.

[8] Hughes, T.J.R., and Brezzi, F., "On Drilling Degrees of Freedom", Computer Methods in Applied Mechanics and Engineering, Vol. 72, 1989, pp. 105-121.

[9] Ibrahimbegovic, A., Taylor, R.L., and Wilson, E.L., "A Robust Quadrilateral Membrane Finite Element with Drilling Degrees of Freedom", International Journal for Numerical Methods in Engineering, Vol. 30, 1990, pp. 445-457.

[10] Ibrahimbegovic, A., and Wilson, E.L., "A Unified Formulation for Triangular and Quadrilateral Flat Shell Finite Elements with Six Nodal Degrees of Freedom", International Journal for Numerical Methods in Engineering, Vol. 7, 1991, pp. 1-9.

[11] Iura, M., and Atluri, S.N., "Formulation of a Membrane Finite Element with Drilling Degrees of Freedom", Computational Mechanics, Vol. 9, 1992, pp. 417428.

[12] Robinson, J., "Four-Node Quadrilateral Stress Membrane Element with Rotational Stiffness", International Journal for Numerical Methods in Engineering, Vol. 16, 1980, pp. 1567-1569. 
[13] Irons, B.M., and Ahmad, S., Techniques of Finite Elements, Ellis Horwood Ltd., Chichester, West Sussex, 1980.

[14] Cook, R.D., "On the Allman Triangle and a Related Quadrilateral Element", Computers and Structures, Vol. 22, 1986, pp. 1065-1067.

[15] Allman, D.J., "A Quadrilateral Finite Element Including Vertex Rotation for Plane Elasticity Analysis", International Journal for Numerical Methods in Engineering, Vol. 26, 1988, pp. 717-730.

[16] MacNeal, R.H., and Harder, R.L., "A Refined Four-Noded Membrane Element with Rotational Degrees of Freedom", Computers and Structures, Vol. 28, 1988, pp. 75-84.

[17] Yunus, S.H., Saigal, S., and Cook, R.D., "On Improved Hybrid Finite Elements with Rotational Degrees of Freedom", International Journal for Numerical Methods in Engineering, Vol. 28, 1989, pp. 785-800.

[18] Aminpour, M.A., "An Assumed-Stress Hybrid 4-Node Shell Element with Drilling Degrees of Freedom", International Journal for Numerical Methods in Engineering, Vol. 33, 1992, pp. 19-38.

[19] Aminpour, M.A., "Direct Formulation of a Hybrid 4-Node Shell Element with Drilling Degrees of Freedom", International Journal for Numerical Methods in Engineering, Vol. 35, 1992, pp. 997-1013.

[20] Pian, T.H.H., Chen, D.P., and Kang, D., "A New Formulation of Hybrid/Mixed Finite Element", Computers and Structures, Vol. 16, 1983, pp. 81-87.

[21] Pian, T.H.H., "Derivation of Element Stiffness Matrices by Assumed Stress Distribution", AIAA Journal, Vol. 2, 1964, pp. 1333-1336.

[22] Pian, T.H.H., and Chen, D.P., "On the Suppression of Zero Energy Deformation Modes", International Journal for Numerical Methods in Engineering, Vol. 19, 1983, pp. 1741-1752.

[23] Kang, D., Hybrid Stress Finite Element Method, Ph.D. Dissertation, Massachusetts Institute of Technology, Cambridge, MA, 1986.

[24] Brezzi, F., and Fortin, M., Mixed and Hybrid Finite Element Methods, SpringerVerlag, New York, 1991.

[25] Fraejis de Veubeke, B., "Displacement and Equilibrium Models in the Finite Element Method", Zienkiewicz, O.C., and Holister, G.S., (Editors), Stress Analysis, Wiley, London, 1965, pp. 145-197.

[26] Stewart, C.B., "The Computational Structural Mechanics Testbed User's Manual", NASA TM-100644 (updated), 1990. 
[27] Stanley, G.M., and Nour-Omid, S.,"The Computational Structural Mechanics Testbed Generic Structural Element Processor Manual", NASA CR-181728, 1990.

[28] MacNeal, R.H., and Harder, R.L., "A Proposed Standard Set of Problems to Test Finite Element Accuracy", Finite Elements in Analysis and Design, Vol. 1, 1985 , pp. 3-20.

[29] Timoshenko, S.P., and Goodier, J.N., Theory of Elasticity, Third Edition, McGraw-Hill, New York, 1970.

[30] Scordelis, L.O., and Lo, K.S., "Computer Analysis of Cylindrical Shells", Journal of American Concrete Institute, Vol. 61, 1969, pp. 539-561.

[31] Timoshenko, S.P., and Woinowsky-Krieger, S., Theory of Plates and Shells, Second Edition, McGraw-Hill, New York, 1959.

[32] Hartung, R.F., and Ball, R.E., "A Comparison of Several Computer Solutions to Three Structural Shell Analysis Problems", AFDL TR-73-15, Air Force Flight Dynamics Laboratory, Dayton, OH, April 1973.

[33] Park, K.C., and Stanley, G.M., "A Curved $C^{0}$ Shell Element Based on Assumed Natural-Coordinate Strains", ASME Journal of Applied Mechanics, Vol. 108, 1986, pp. 278-290.

[34] Stewart, C.B., "The Computational Structural Mechanics Testbed Procedures Manual", NASA CR-100646, 1990.

[35] Forsberg, K., "Influence of Boundary Conditions on the Modal Characteristics of Thin Cylindrical Shells", AIAA Journal, Vol. 2, 1964, pp. 2150-2157.

[36] Almroth, B.O., Brogan, F.A., and Stanley, G.M., "Structural Analysis of General Shells", Vol. II, User's Introduction for STAGSC-1 Computer Code, Report Number: LMSC-D633873, Lockheed Palo Alto Research Laboratory, Palo Alto, CA, December 1982.

[37] Aminpour, M.A., "Assessment of SPAR Elements and Formulation of Some Basic 2-D and 3-D Elements for Use with Testbed Generic Element Processor", Proceedings of NASA Workshop on Computational Structural Mechanics - 1987, NASA CP-10012, Part 2, Nancy P. Sykes (Editor), 1989, pp. 653-682. 


\section{Appendix A}

EX47(ES1): A $C^{0}$, isoparametric, assumed natural-coordinate strain element, developed by Park and Stanley [33]. The element is not invariant with respect to reference coordinates and does not pass the patch test. This element will be referred to as 4_ANS.

QUAD4: A 4-node isoparametric shell element with selective reduced integration [28]. This element is available in MSC/NASTRAN. Q4S, an improved version of QUAD4, contains the drilling degree of freedom [16].

E410(ES5): A $C^{1}$, incompatible, displacement based element with drilling degrees of freedom. The element kernels were extracted from the STAGS computer code (Almroth et al.[36]). The element uses cubic interpolation for the displacement field. This element is not invariant and does not pass the patch test. This element will be referred to as 4_STG.

EX43(ES4): A $C^{0}$, isoparametric, assumed-stress hybrid shell element with no drilling degree of freedom. This element is invariant with respect to reference coordinates and passes the patch test. This element was developed by Aminpour[37]. This element will be referred to as 4_HYB.

AQR8(ES8): An assumed-stress hybrid shell element with drilling degrees of freedom, developed by Aminpour [18]. The formulation is based on Hellinger-Reissner variational principle. The 4-node element is obtained from an "internal" 8node element.

AQD4(ES8): An assumed-stress hybrid shell element with drilling degrees of freedom, developed by Aminpour [19]. The formulation is based on modified complementary energy principle and the stress field is expanded in Cartesian coordinates. 


\section{List of Tables}

Table

I. MacNeal-Harder straight cantilever beams.

II. MacNeal-Harder twisted cantilever beam.

III. MacNeal-Harder curved cantilever beam.

IV. Scordelis-Lo roof.

V. Morley's spherical shell.

VI. Linear buckling analysis of the pear-shaped cylinder.

VII. Linear buckling analysis of the rectangular plate under uniaxial compression.

VIII. Linear buckling analysis of the rectangular plate under biaxial compression.

IX. Linear buckling analysis of the rectangular plate under uniform shear.

$\mathrm{X}$. Linear free vibration analysis of the rectangular plate.

XI. Linear free vibration analysis of the cylindrical shell. 
Table I. MacNeal-Harder straight cantilever beams.

\begin{tabular}{|l|l|l|l|l|l|l|l|}
\hline \multicolumn{8}{|c|}{ A : Extension, B : In-plane Shear, C : Out-of-plane Shear, D : Twist } \\
\hline Load & 4_ANS & QUAD4 & 4_STG & 4_HYB & AQR8 & AQD4 & A4S1 \\
\hline \hline \multicolumn{7}{|c|}{ Rectangular-Shaped Elements } \\
\hline A & 0.995 & 0.995 & 0.994 & 0.996 & 0.998 & 0.998 & 0.998 \\
B & 0.904 & $0.904^{*}$ & 0.915 & 0.993 & 0.993 & 0.993 & 0.993 \\
C & 0.980 & 0.986 & 0.986 & 0.981 & 0.981 & 0.981 & 0.981 \\
D & 0.856 & $0.941^{* *}$ & 0.680 & 1.023 & 1.011 & 1.011 & 1.009 \\
\hline \multicolumn{7}{|c|}{ Trapezoidal-Shaped Elements } \\
\hline A & 0.761 & 0.996 & 0.991 & 0.999 & 0.998 & 0.998 & 0.998 \\
B & 0.305 & $0.071^{*}$ & 0.813 & 0.052 & 0.986 & 0.986 & 0.986 \\
C & 0.763 & 0.968 & 0.925 & 0.075 & 0.965 & 0.965 & 0.969 \\
D & 0.843 & $0.951^{* *}$ & 0.683 & 1.034 & 1.029 & 1.009 & 1.007 \\
\hline \multicolumn{7}{|c|}{ Parallelogram-Shaped Elements } \\
\hline A & 0.966 & 0.996 & 0.989 & 0.999 & 0.998 & 0.998 & 0.998 \\
B & 0.324 & $0.080^{*}$ & 0.794 & 0.632 & 0.977 & 0.972 & 0.977 \\
C & 0.939 & 0.977 & 0.991 & 0.634 & 0.980 & 0.980 & 0.980 \\
D & 0.798 & $0.945^{* *}$ & 0.677 & 1.166 & 1.159 & 1.010 & 1.007 \\
\hline
\end{tabular}

* Q4S results for these cases are $0.993,0.988$, and 0.986 , respectively.

** These results were normalized with respect to 0.03208 in reference [28]. However, all the other results for twist are normalized with respect to 0.03406 as reported in Timoshenko and Goodier [29].

Table II. MacNeal-Harder twisted cantilever beam.

\begin{tabular}{|c|c|l|c|c|c|c|c|}
\hline Load & 4_ANS & QUAD4 & 4_STG & 4_HYB & AQR8 & AQD4 & A4S1 \\
\hline \hline In-plane shear & 1.357 & 0.993 & 1.054 & 1.361 & 0.991 & 0.991 & 0.991 \\
Out-of-plane shear & 1.293 & 0.985 & 1.173 & 1.359 & 1.093 & 1.093 & 1.093 \\
\hline
\end{tabular}

Table III. MacNeal-Harder curved cantilever beam.

\begin{tabular}{|c|c|l|c|c|c|c|c|}
\hline Load & 4_ANS & QUAD4 & 4_STG & 4_HYB & AQR8 & AQD4 & A4S1 \\
\hline \hline In-plane shear & 0.929 & 0.833 & 0.938 & 0.888 & 0.997 & 0.996 & 0.997 \\
Out-of-plane shear & 0.935 & 0.951 & 0.887 & 0.925 & 0.956 & 0.956 & 0.970 \\
\hline
\end{tabular}


Table IV. Scordelis-Lo roof.

\begin{tabular}{|c|c|c|c|c|c|c|c|}
\hline Mesh & 4_ANS & QUAD4 & 4_STG & 4_HYB & AQR8 & AQD4 & A4S1 \\
\hline \hline $2 \times 2$ & 1.387 & 1.376 & 1.384 & 1.459 & 1.218 & 1.218 & 1.222 \\
$4 \times 4$ & 1.039 & 1.050 & 1.049 & 1.068 & 1.021 & 1.021 & 1.022 \\
$6 \times 6$ & 1.011 & 1.018 & 1.015 & 1.028 & 1.006 & 1.006 & 1.007 \\
$8 \times 8$ & 1.005 & 1.008 & 1.005 & 1.017 & 1.003 & 1.003 & 1.003 \\
$10 \times 10$ & 1.003 & 1.004 & 1.001 & 1.011 & 1.001 & 1.001 & 1.001 \\
\hline
\end{tabular}

Table V. Morley's Spherical Shell.

\begin{tabular}{|c|c|c|c|c|c|c|c|}
\hline Mesh & 4_ANS & QUAD4 & 4_STG & 4_HYB & AQR8 & AQD4 & A4S1 \\
\hline \hline $2 \times 2$ & 0.968 & 0.972 & 0.338 & 1.032 & 0.382 & 0.381 & 0.425 \\
$4 \times 4$ & 1.018 & 1.024 & 0.519 & 1.093 & 0.227 & 0.226 & 0.231 \\
$6 \times 6$ & 1.001 & 1.013 & 0.841 & 1.060 & 0.432 & 0.432 & 0.433 \\
$8 \times 8$ & 0.995 & 1.005 & 0.949 & 1.040 & 0.681 & 0.680 & 0.682 \\
$10 \times 10$ & 0.993 & 1.001 & 0.978 & 1.027 & 0.835 & 0.835 & 0.838 \\
$12 \times 12$ & 0.992 & 0.998 & 0.988 & 1.020 & 0.914 & 0.914 & 0.916 \\
\hline
\end{tabular}

Table VI. Linear buckling analysis of the pear-shaped cylinder.

\begin{tabular}{|c|c|c|c|c|c|}
\hline \multirow{2}{*}{ Modes } & \multirow{2}{*}{$\lambda_{c r}^{*}$} & \multicolumn{4}{|c|}{$\lambda_{c r} / \lambda_{c r}^{*}$} \\
\cline { 3 - 6 } & 9_ANS & 4_ANS & 4_STG & 4_HYB & A4S1 \\
\hline \hline 1 & 4.393 & 1.021 & 1.002 & 1.016 & 1.013 \\
2 & 6.483 & 1.032 & 1.013 & 1.027 & 1.016 \\
\hline
\end{tabular}

Table VII. Linear buckling analysis of the rectangular plate under uniaxial compression.

\begin{tabular}{|c|c|c|c|c|c|c|}
\hline & & $N_{y}^{*}$ & \multicolumn{4}{|c|}{$N_{y} / N_{y}^{*}$} \\
\cline { 4 - 7 } Modes & $n$ & Analytical & 4_ANS & 4_STG & 4_HYB & A4S1 \\
\hline \hline 1 & 1 & 523.04 & 1.013 & 0.991 & 1.008 & 0.998 \\
2 & 3 & 874.89 & 1.044 & 0.988 & 1.023 & 1.001 \\
\hline
\end{tabular}


Table VIII. Linear buckling analysis of the rectangular plate under biaxial compression $\left(N_{x}=N_{y}=N\right)$.

\begin{tabular}{|c|c|c|c|c|c|c|c|}
\hline \multirow[b]{2}{*}{ Modes } & \multirow[b]{2}{*}{$m$} & \multirow[b]{2}{*}{$n$} & \multirow{2}{*}{$\begin{array}{c}N^{*} \\
\text { Analytical }\end{array}$} & \multicolumn{4}{|c|}{$N / N^{*}$} \\
\hline & & & & 4_ANS & 4_STG & 4_HYB & $\mathrm{A} 4 \mathrm{~S} 1$ \\
\hline 1 & 1 & 1 & $\overline{188.29}$ & 1.010 & 0.995 & 1.005 & 0.99 \\
\hline 2 & 1 & 3 & 730.58 & 1.047 & 0.981 & 1.027 & 1.00 \\
\hline 3 & 3 & 1 & 1152.36 & 1.153 & 0.998 & 1.095 & 1.020 \\
\hline
\end{tabular}

Table IX. Linear buckling analysis of the rectangular plate under uniform shear.

\begin{tabular}{|c|c|c|c|c|c|}
\hline & $N_{x y}^{*}$ & \multicolumn{4}{|c|}{$N_{x y} / N_{x y}^{*}$} \\
\cline { 3 - 6 } Modes & Analytical & 4_ANS & 4_STG & 4_HYB & A4S1 \\
\hline \hline 1 & 3629.69 & 1.284 & 0.930 & 1.213 & 0.989 \\
\hline
\end{tabular}

Table X. Linear free vibration analysis of the rectangular plate.

\begin{tabular}{|c|c|c|c|c|c|c|}
\hline \multirow{2}{*}{ Modes } & & & \multicolumn{4}{|c|}{ Frequency } \\
\cline { 5 - 7 } & & $n$ & $f^{*}$ & \multicolumn{3}{|c|}{$f / f^{*}$} \\
\cline { 5 - 7 } & & Analytical & 4_ANS & 4_HYB & A4S1 \\
\hline \hline 1 & 1 & 1 & 112.40 & 1.008 & 1.005 & 1.010 \\
2 & 1 & 3 & 436.10 & 1.036 & 1.026 & 1.037 \\
3 & 3 & 1 & 687.80 & 1.111 & 1.083 & 1.087 \\
\hline
\end{tabular}

Table XI. Linear free vibration analysis of the cylindrical shell.

\begin{tabular}{|c|c|c|c|c|c|c|}
\hline \multicolumn{7}{|c|}{ Number of circumferential waves $=6$} \\
\hline \multirow[b]{4}{*}{ Mesh } & \multirow{2}{*}{\multicolumn{3}{|c|}{$\begin{array}{c}\text { Symmetric Mode } 1 \\
\text { Theoretical }[35] \\
\omega_{T}^{2}=0.633 \times 10^{5} \\
\end{array}$}} & \multirow{2}{*}{\multicolumn{3}{|c|}{$\begin{array}{c}\text { Anti-symmetric Mode } 1 \\
\text { Theoretical }[35] \\
\omega_{T}^{2}=0.581 \times 10^{5}\end{array}$}} \\
\hline & & & & & & \\
\hline & \multicolumn{3}{|c|}{$\omega^{2} / \omega_{T}^{2}$} & \multicolumn{3}{|c|}{$\omega^{2} / \omega_{T}^{2}$} \\
\hline & 4_ANS & 4_HYB & A4S1 & 4_ANS & 4_HYB & A4S1 \\
\hline & 1.022 & 1.013 & 1.0 & 1.028 & 1.010 & 1.026 \\
\hline & 1.008 & 1.000 & 1.009 & 1.009 & 0.995 & 1.012 \\
\hline & .002 & 0.995 & 1.005 & 1.002 & 0.988 & 1.005 \\
\hline
\end{tabular}




\section{List of Figures}

Figure

1. The patch test.

2. MacNeal-Harder straight cantilever beams.

3. MacNeal-Harder twisted cantilever beam.

4. MacNeal-Harder curved cantilever beam.

5. Scordelis-Lo roof.

6. Morley's spherical shell.

7. Convergence study for the isotropic square plate; $a=4, h=0.001$, $P=100, E=10^{7}, \nu=0.3$.

8. The effect of mesh distortion (for a $7 \times 7$ mesh).

9. The effect of thickness change (for a $7 \times 7$ mesh).

10. The pear-shaped cylinder.

11. The variation of radial displacements along the half-cylinder circumference.

12. The variation of axial stress resultant along the half-cylinder circumference.

13. Buckling mode shapes for the pear-shaped cylinder.

14. Linear buckling analysis of the rectangular plate under (a) uniaxial compression, (b) biaxial compression, and (c) uniform shear; length along the $\mathrm{X}$-direction $=15$, length along the $\mathrm{Y}$-direction $=20, E=30 \times 10^{6}, \nu=0.3$.

15. Convergence of the critical load for the axially compressed cylinder; $E=10^{7}, \nu=0.3, R=36, L=100, l=10, t=0.125, \theta=15^{\circ}$.

16. Mode shapes corresponding to the lowest two critical loads for the axially compressed cylinder.

17. Vibration mode shapes for the cylindrical shell; $E=10^{7}, \nu=0.3$, $\rho=0.1, R=10, t=0.1, h=5$. 


\begin{tabular}{|ccc|}
\hline \multicolumn{3}{|c|}{ Location of Nodes } \\
\hline Node & $\mathrm{x}$ & $\mathrm{y}$ \\
\hline \hline 1 & 0.04 & 0.02 \\
2 & 0.18 & 0.03 \\
3 & 0.16 & 0.08 \\
4 & 0.08 & 0.08 \\
\hline
\end{tabular}

\begin{tabular}{|c|c|}
\hline \multicolumn{2}{|c|}{$\mathrm{a}=0.12, \mathrm{~b}=0.24, \mathrm{t}=0.001, E=10^{8}, \nu=0.25$} \\
\hline Boundary Conditions & Theoretical Solution \\
\hline \multicolumn{2}{|c|}{ Membrane Patch Test } \\
\hline $\begin{array}{l}u=10^{-3}(x+y / 2) \\
v=10^{-3}(x / 2+y)\end{array}$ & $\begin{array}{l}N_{x}=N_{y}=1.333 \\
N_{x y}=0.4\end{array}$ \\
\hline \multicolumn{2}{|c|}{ Bending Patch Test } \\
\hline $\begin{array}{l}w=10^{-3}\left(x^{2}+x y+y^{2}\right) / 2 \\
\theta_{x}=10^{-3}(x / 2+y) \\
\theta_{y}=-10^{-3}(x+y / 2)\end{array}$ & $\begin{array}{l}M_{x}=M_{y}=-1.111 \times 10^{-7} \\
M_{x y}=-3.333 \times 10^{-8}\end{array}$ \\
\hline
\end{tabular}

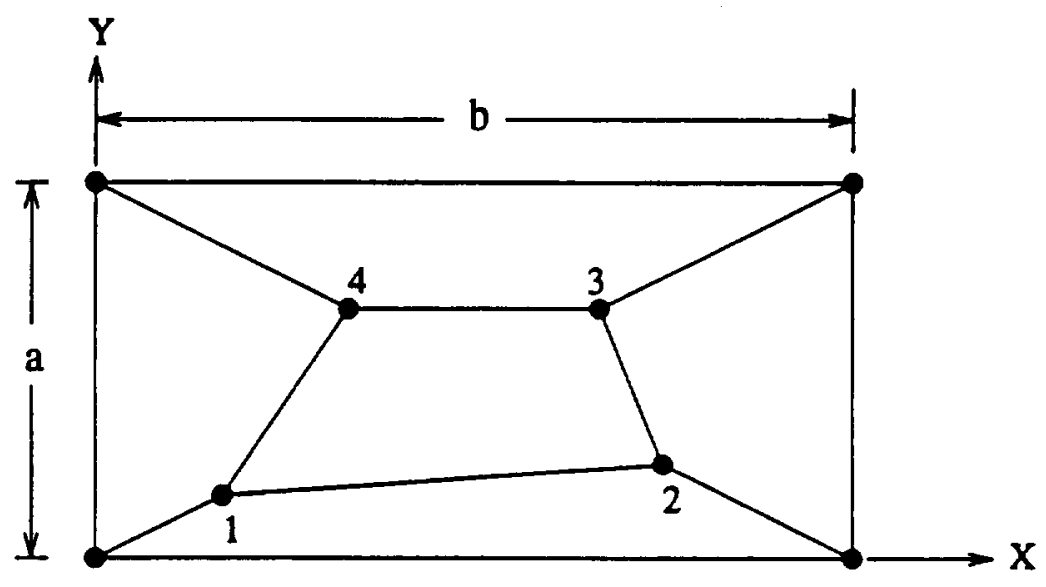

Figure 1. The patch test. 


\begin{tabular}{|c|c|c|}
\hline \multicolumn{3}{|c|}{ Theoretical Solutions } \\
\hline Load Designation & Tip Load Direction & Displacement in the Direction of Load \\
\hline \hline A & Extension & $0.3 \times 10^{-4}$ \\
B & In-Plane Shear & 0.1081 \\
C & Out-of-Plane Shear & 0.4321 \\
D & Twist & 0.03406 \\
\hline
\end{tabular}

\begin{tabular}{|c|c|}
\hline Length & 6.0 \\
height & 0.2 \\
Width & 0.1 \\
Elastic Modulus & $10^{7}$ \\
Poisson's Ratio $(\nu)$ & 0.3 \\
\hline
\end{tabular}

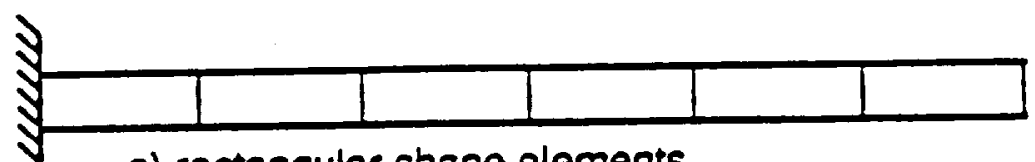

a) rectangular shape elements

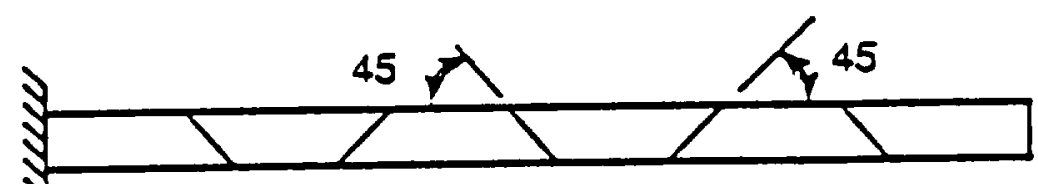

b) trapezoidal shape elements

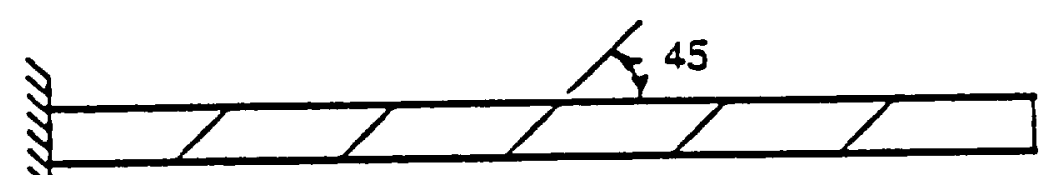

c) parallelogram shape elements

Figure 2. MacNeal-Harder straight cantilever beams. 


\begin{tabular}{|c|c|}
\hline \multicolumn{2}{|c|}{ Theoretical Solutions } \\
\hline Tip Load Direction & Displacement in the Direction of Load \\
\hline \hline In-Plane Shear & 0.005424 \\
Out-of-Plane Shear & 0.001754 \\
\hline
\end{tabular}

\begin{tabular}{|c|c|}
\hline Length & 12.0 \\
Width & 1.1 \\
Depth & 0.32 \\
Elastic Modulus & $29.0 \times 10^{6}$ \\
Poisson's Ratio $(\nu)$ & 0.22 \\
\hline
\end{tabular}

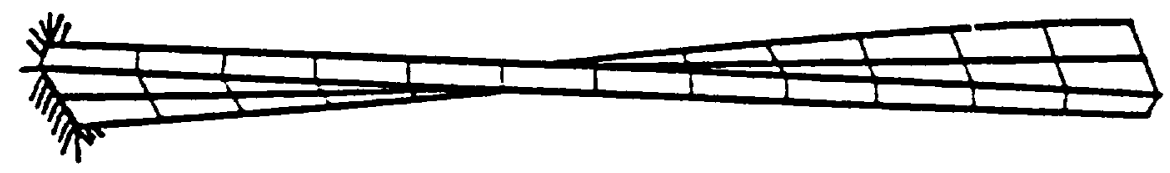

Figure 3. MacNeal-Harder twisted cantilever beam. 


\begin{tabular}{|c|c|}
\hline \multicolumn{2}{|c|}{ Theoretical Solutions } \\
\hline Tip Load Direction & Displacement in the Direction of Load \\
\hline \hline In-Plane Shear & 0.08734 \\
Out-of-Plane Shear & 0.50220 \\
\hline
\end{tabular}

\begin{tabular}{|c|c|}
\hline Inner Radius & 4.12 \\
Outer Radius & 4.32 \\
Depth & 0.10 \\
Elastic Modulus & $10^{7}$ \\
Poisson's Ratio $(\nu)$ & 0.25 \\
\hline
\end{tabular}

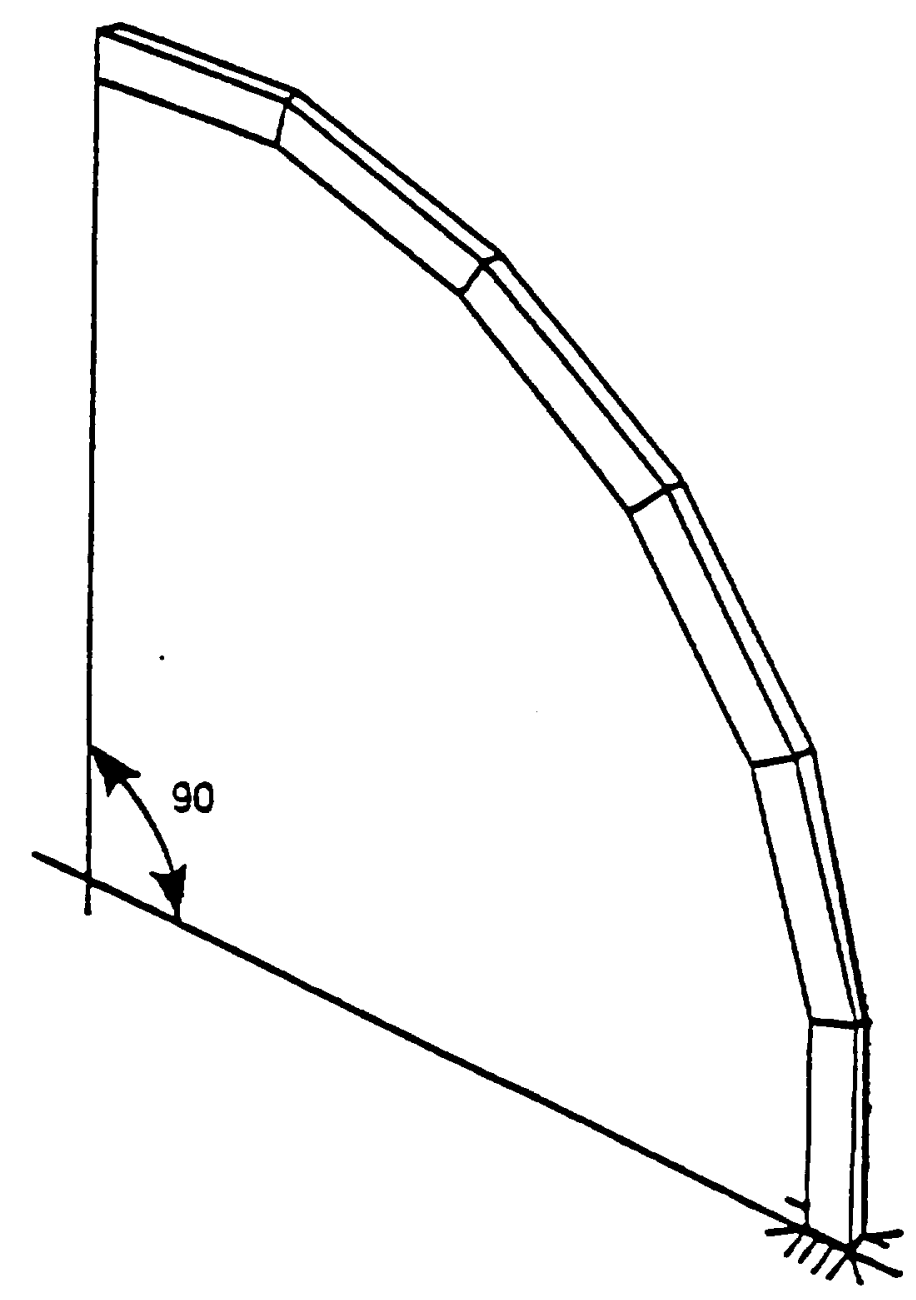

Figure 4. MacNeal-Harder curved cantilever beam. 


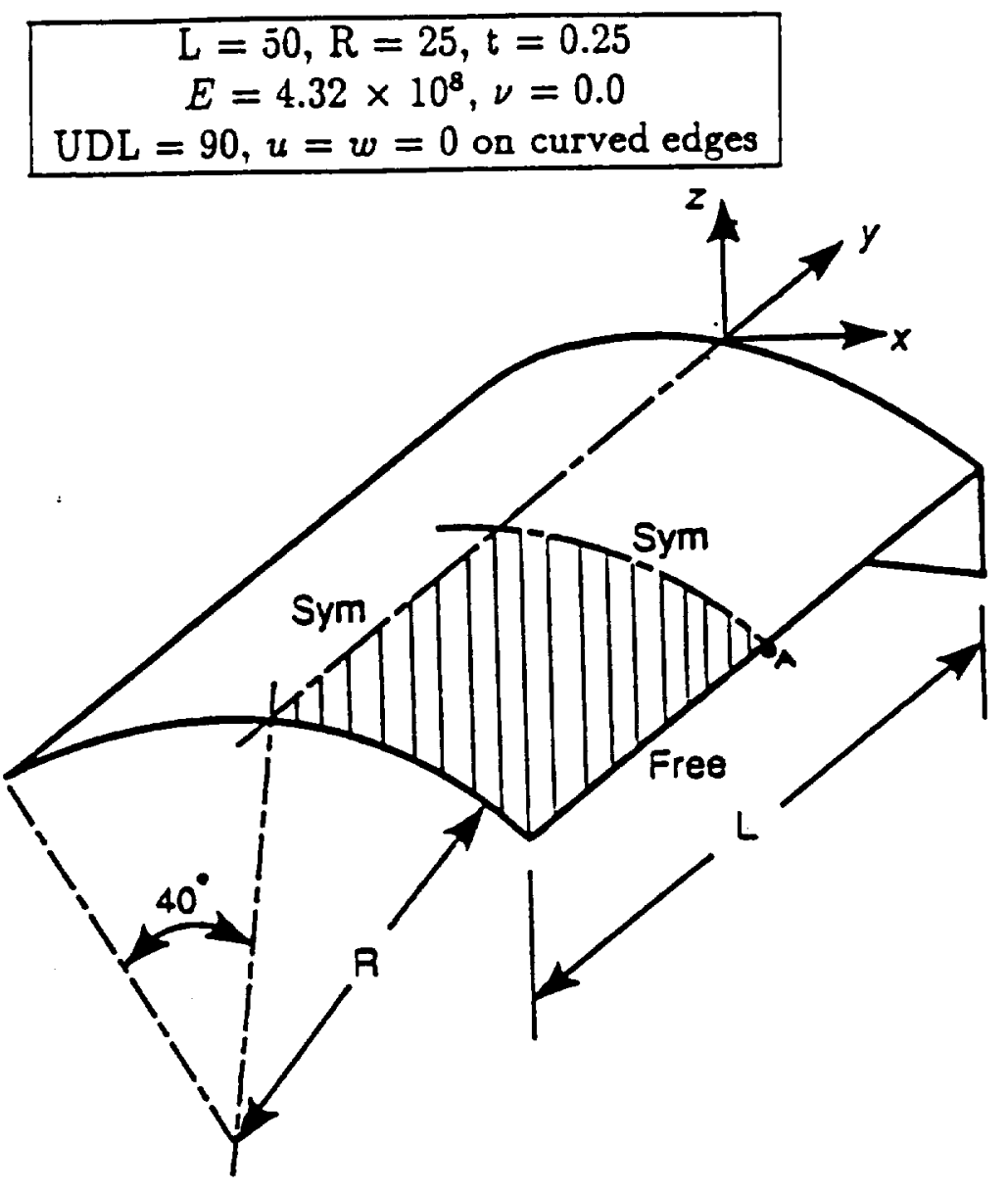

Figure 5. Scordelis-Lo roof. 


$$
\begin{gathered}
\text { Radius }=10 ., \text { Thickness }=0.04 \\
E=6.825 \times 10^{7}, \nu=0.3
\end{gathered}
$$

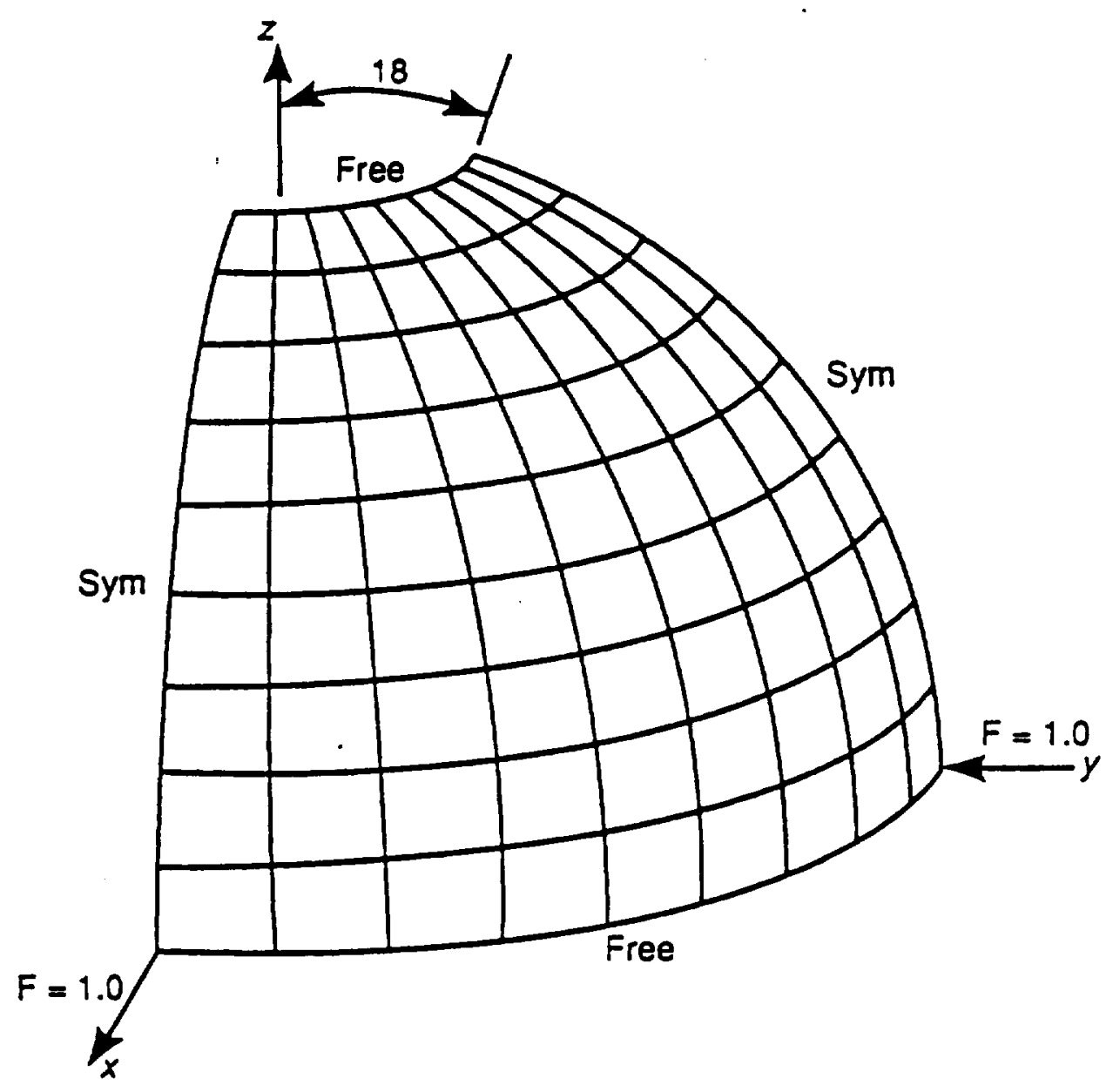

Figure 6. Morley's spherical shell. 


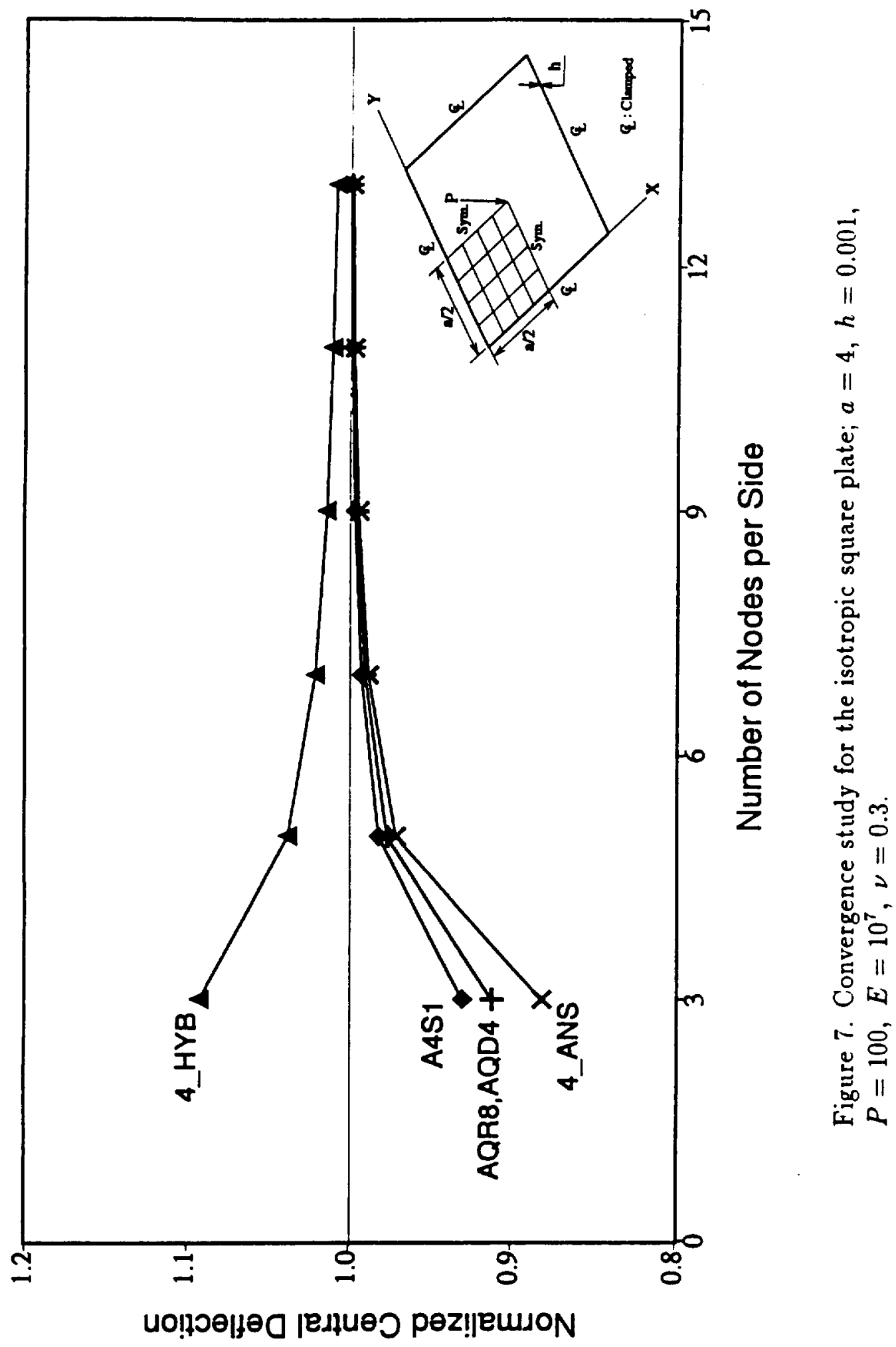




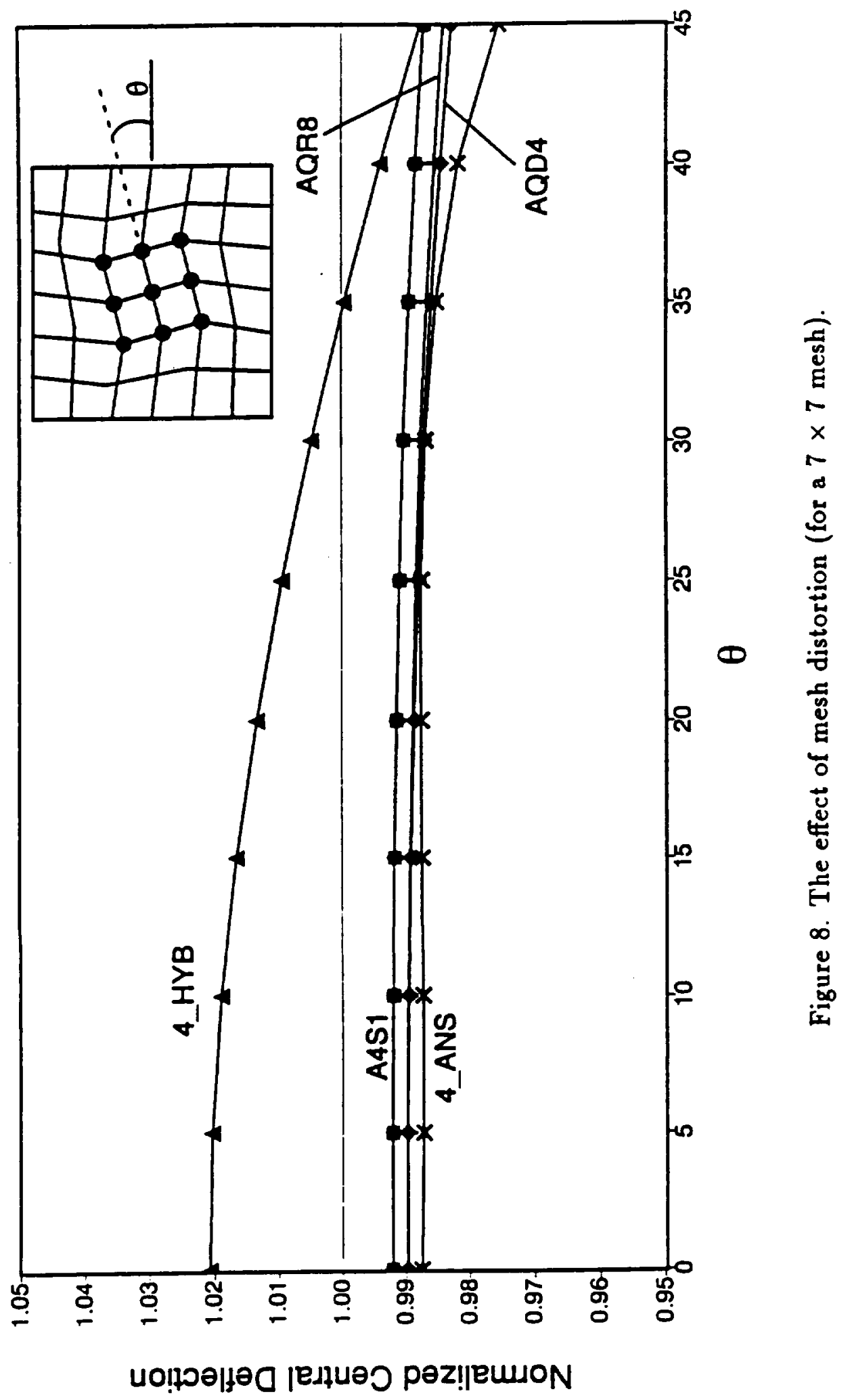




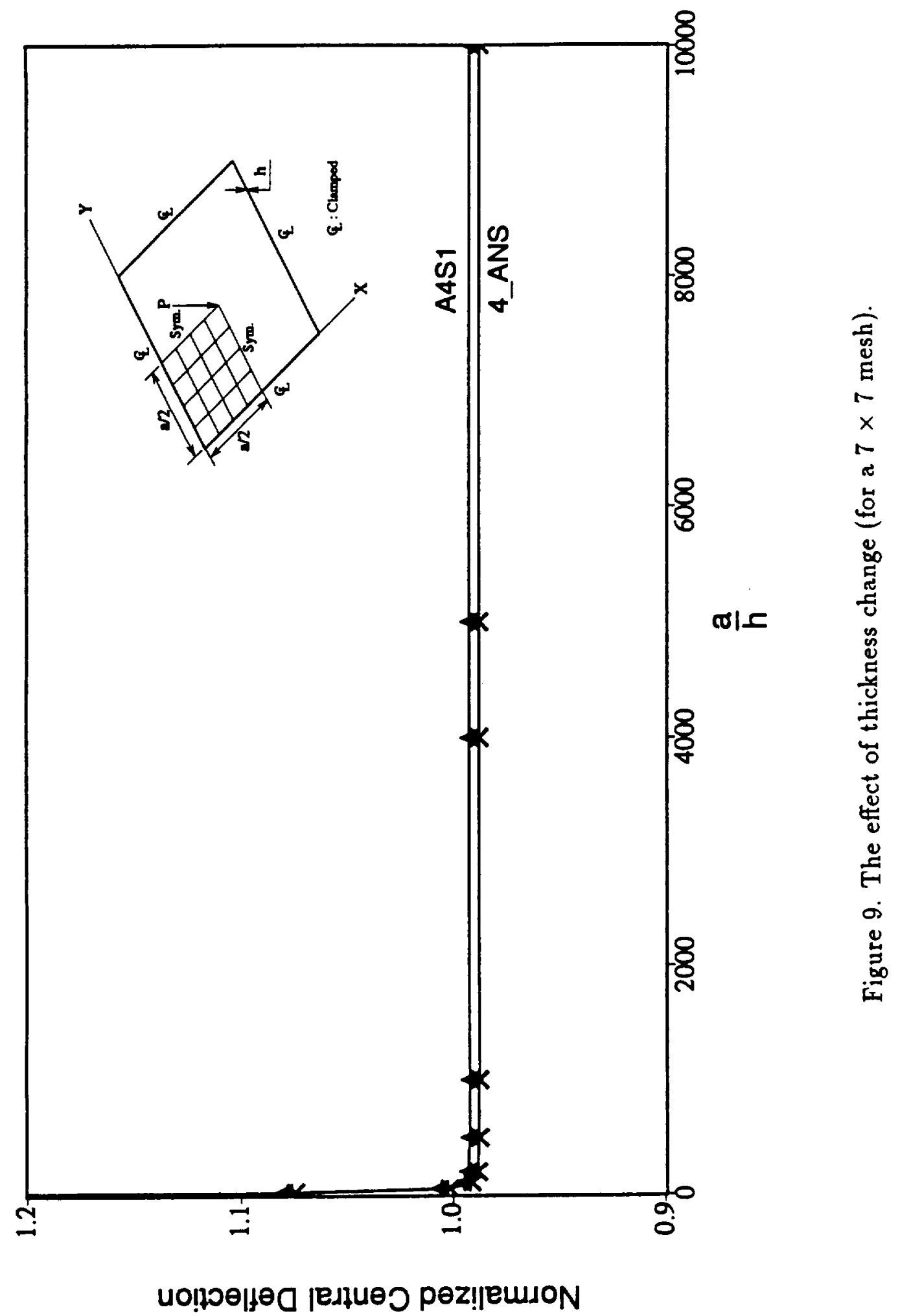




$$
\begin{gathered}
\mathrm{R}=1, \mathrm{~L}=0.8, \mathrm{t}=0.01 \\
E=10^{7}, \nu=0.3
\end{gathered}
$$

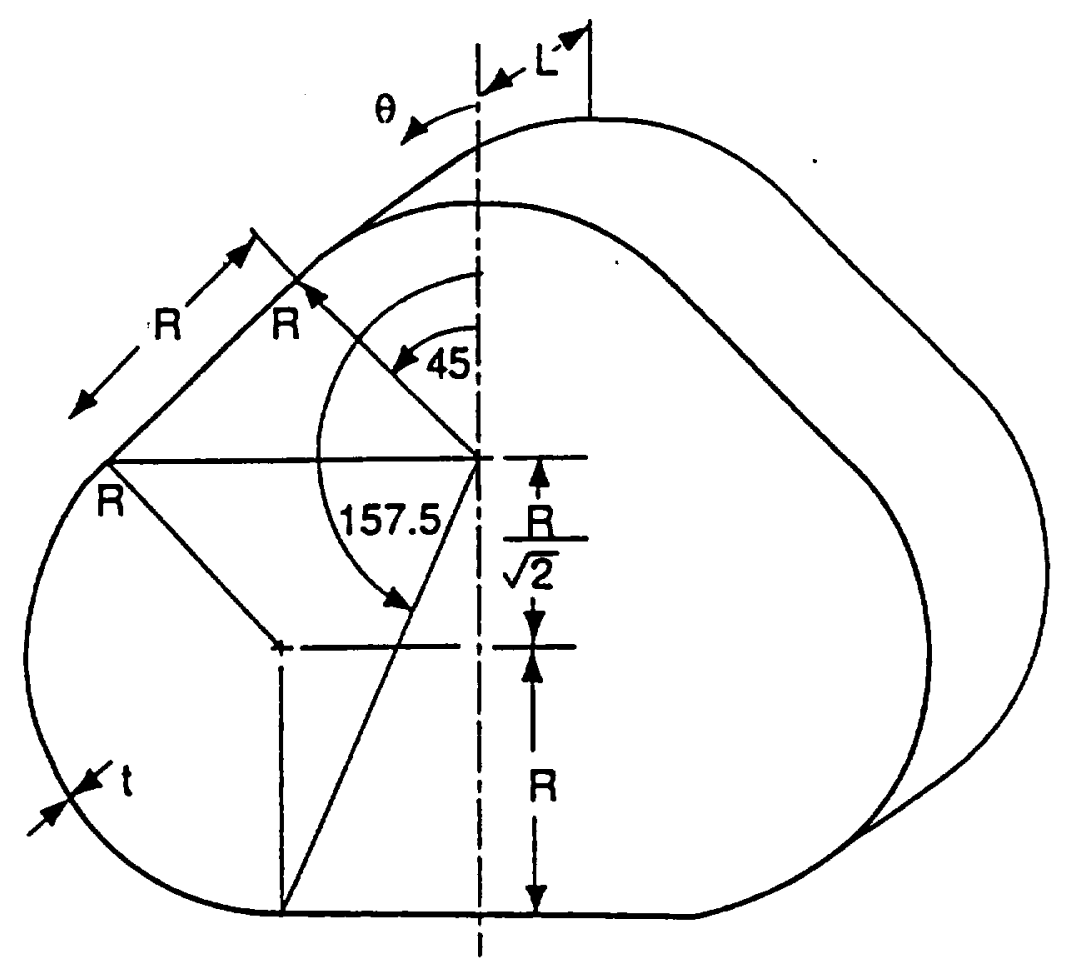

Figure 10. The pear-shaped cylinder. 


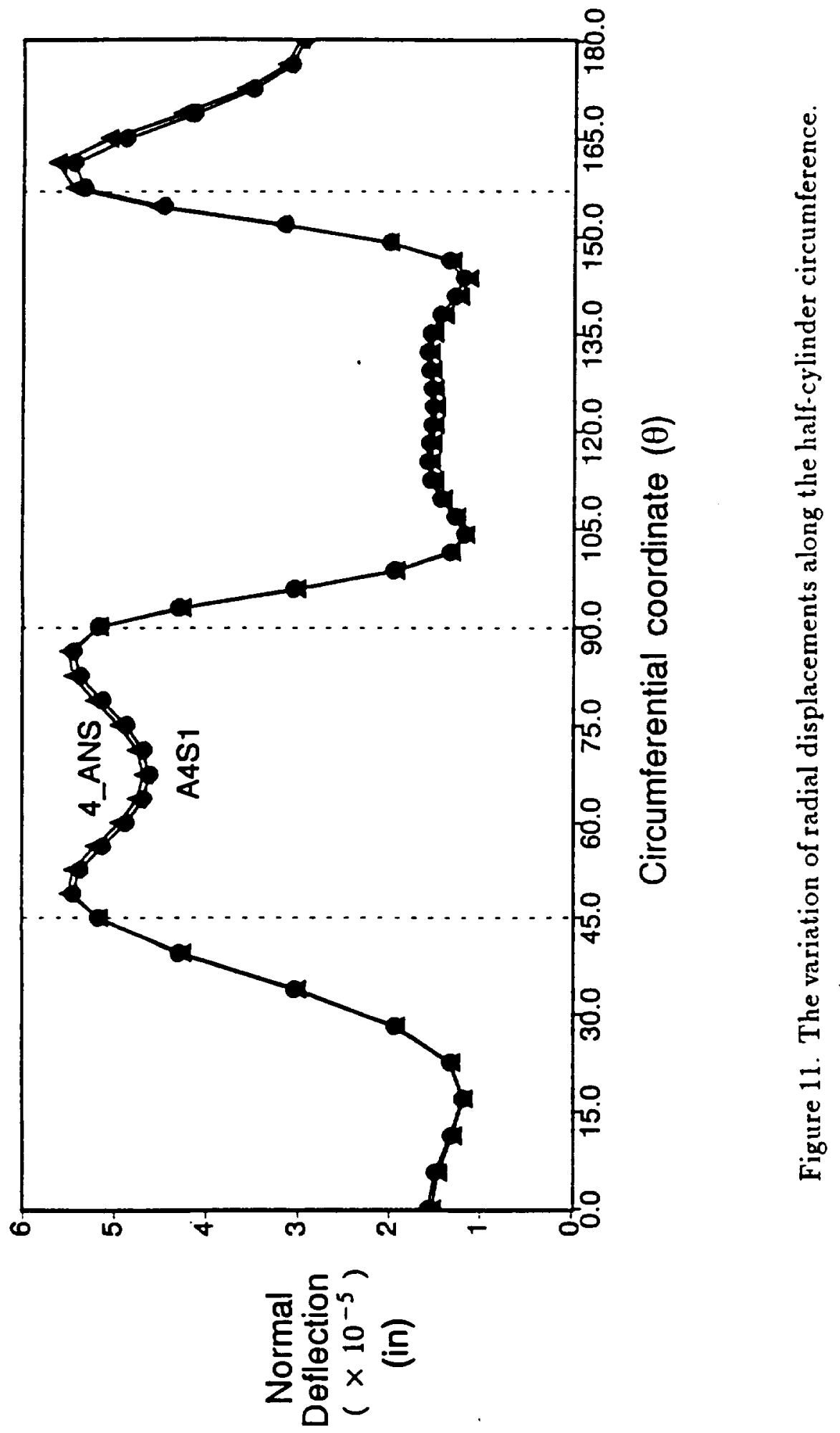




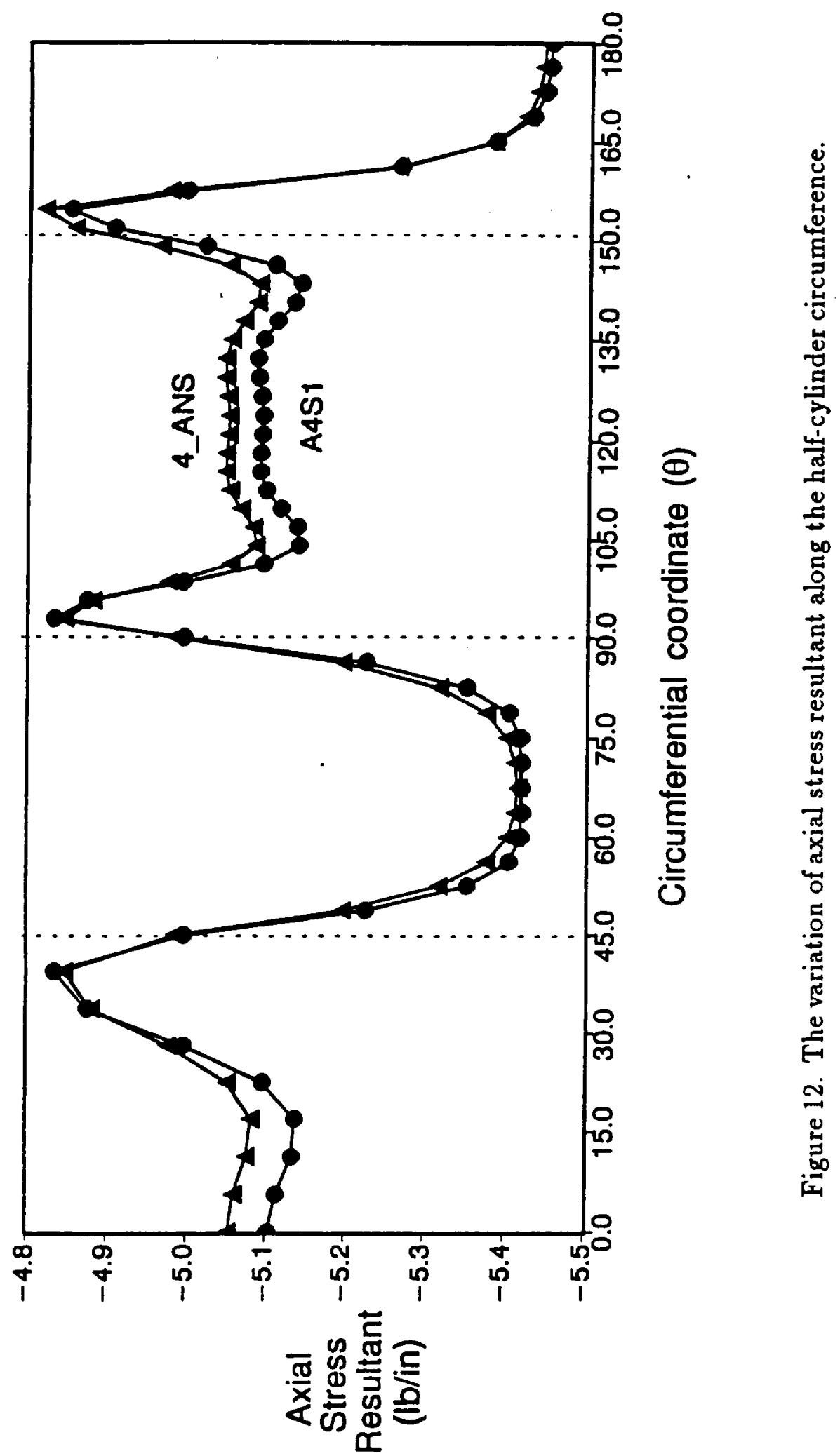




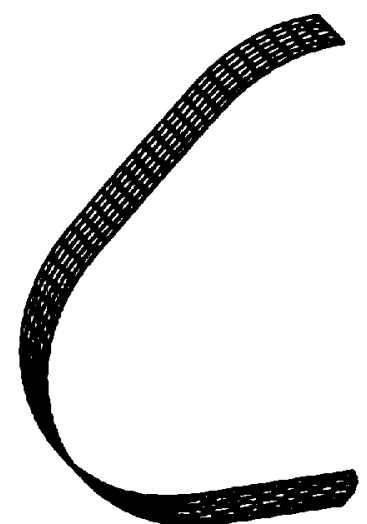

\section{Mode 1}

$N_{y} / N_{y}$ (converged) $=1.013$

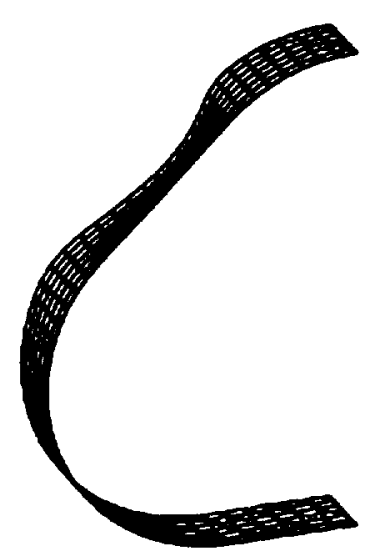

Mode 2

$N_{y} / N_{y}$ (converged) $=1.016$

Figure 13. Buckling mode shapes for the pear-shaped cylinder. 


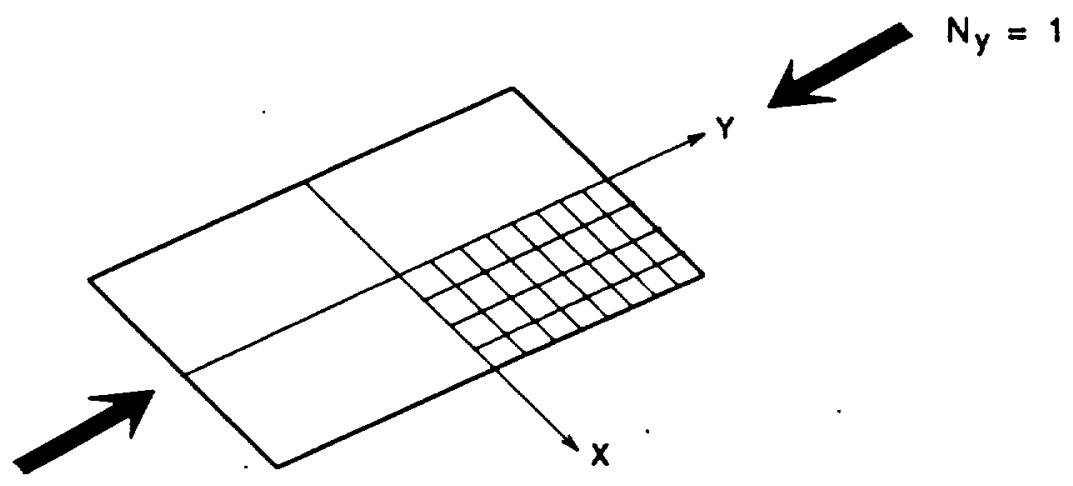

(a)
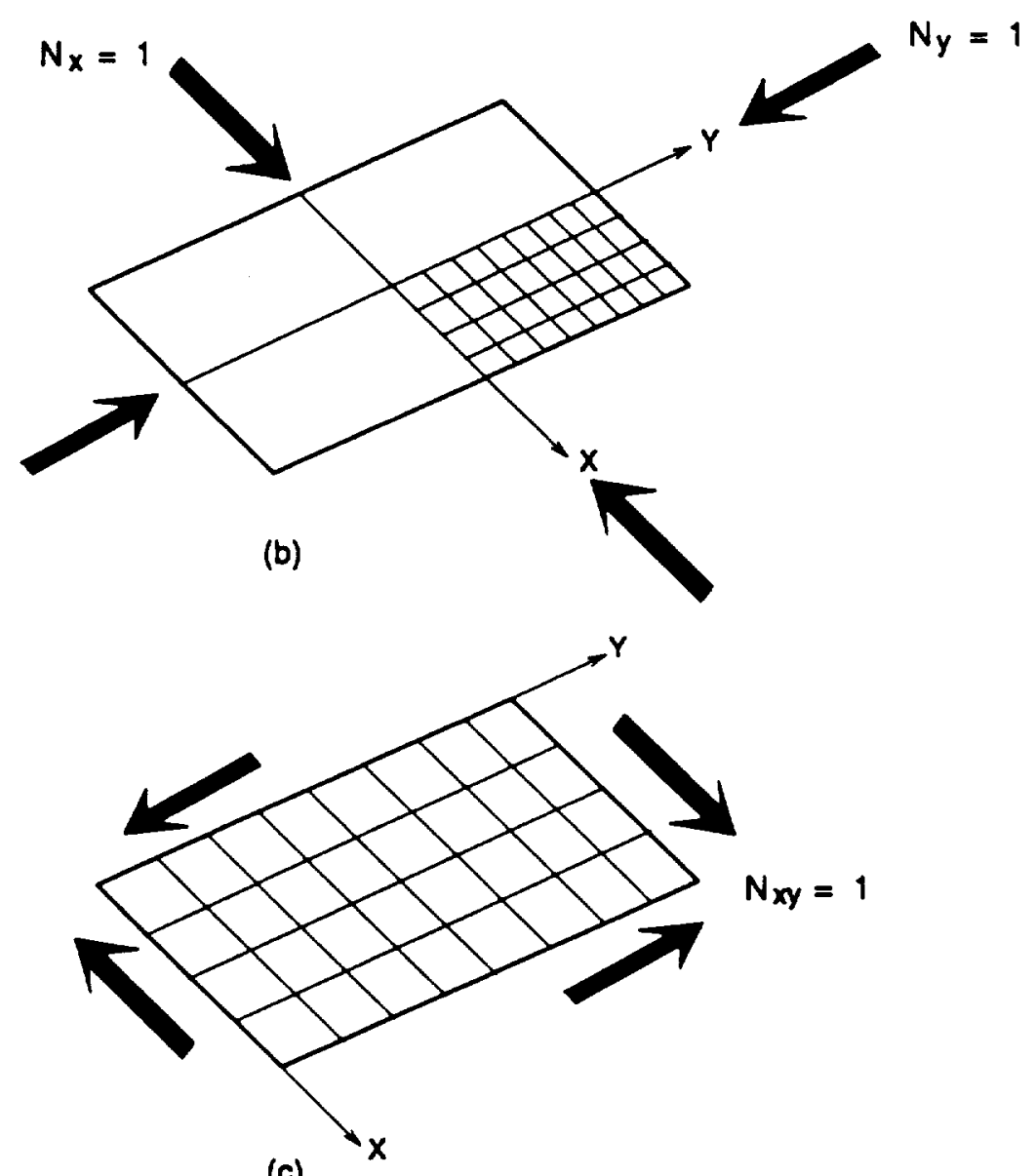

(c)

Figure 14. Linear buckling analysis of the rectangular plate under (a) uniaxial compression, (b) biaxial compression, and (c) uniform shear; length along the $\mathrm{X}$-direction $=15$, length along the Y-direction $=20, E=30 \times 10^{6}, \nu=0.3$. 


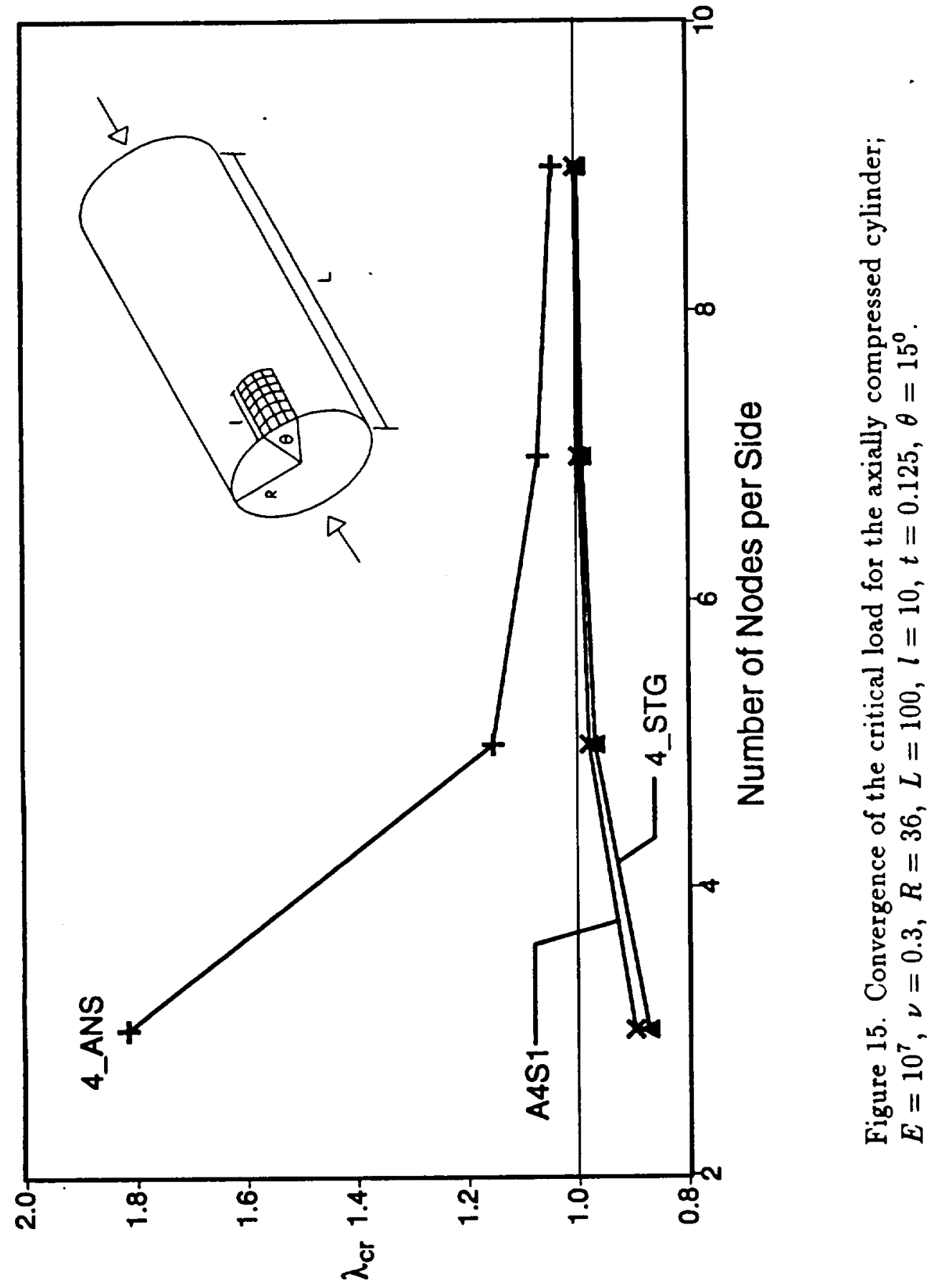




\section{Discretized Model (9 $\times 9)$}
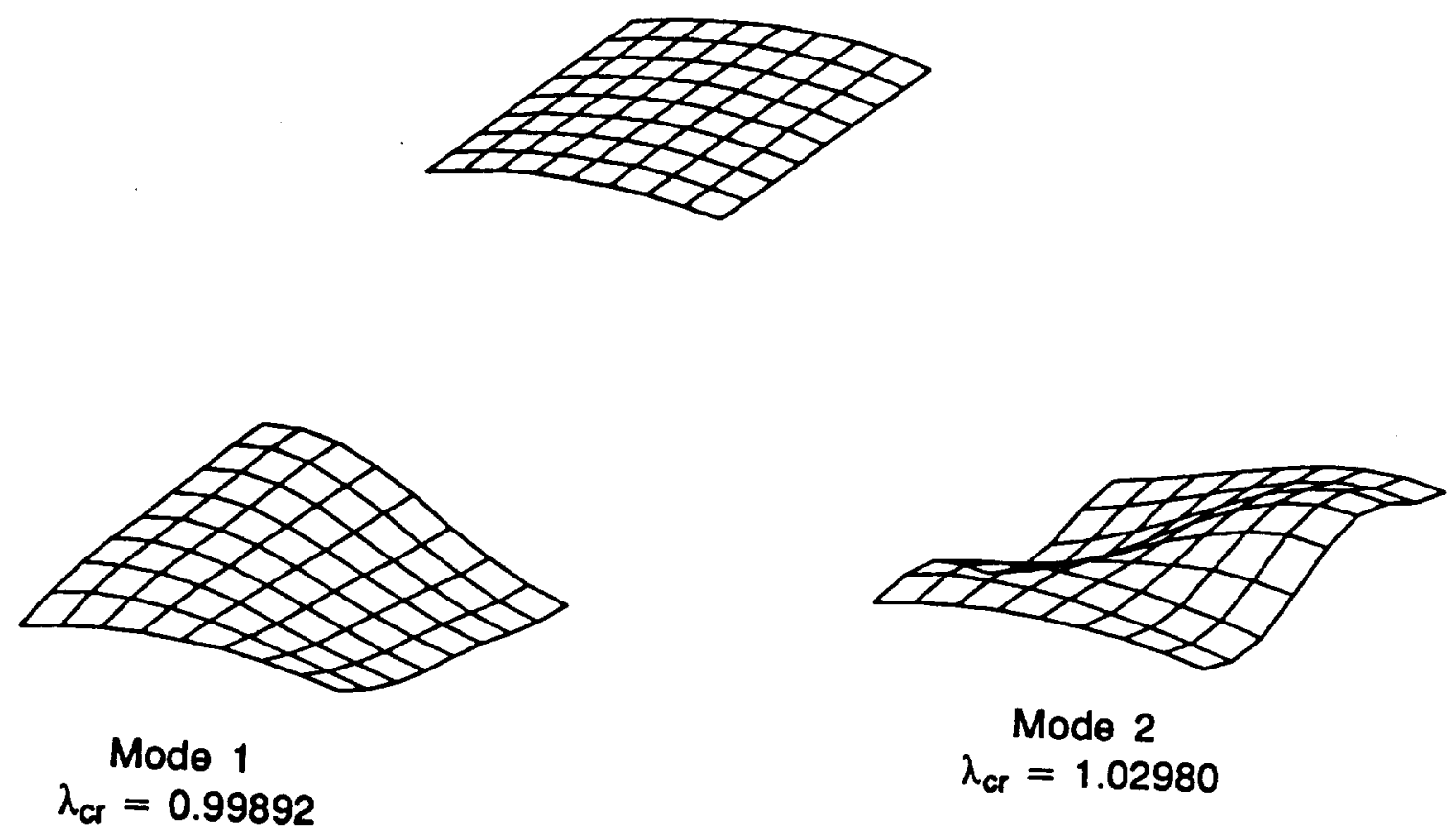

Figure 16. Mode shapes corresponding to the lowest two critical loads for the axially compressed cylinder. 


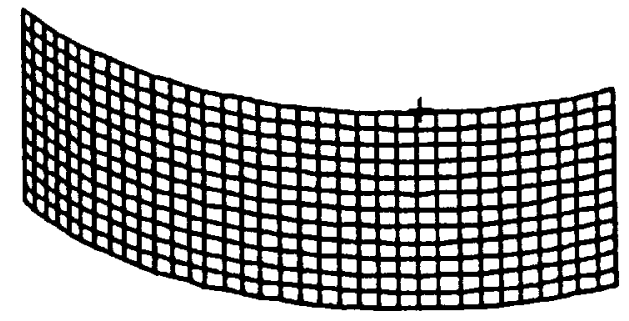

Discretized Model (35 x 13)
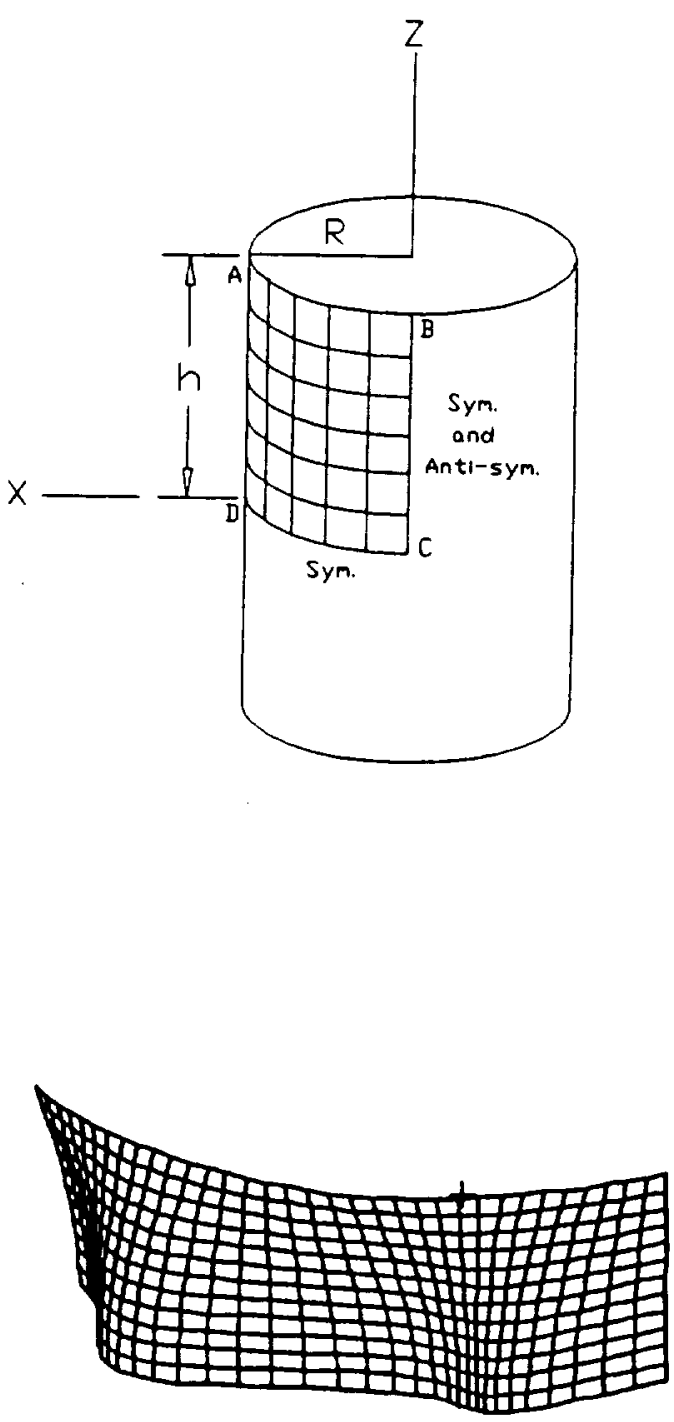

Anti-symmetric Mode 1 $\omega^{2}=0.584 \times 10^{5}$
Symmetric Mode 1

$\omega^{2}=0.636 \times 10^{5}$

Figure 17. Vibration mode shapes for the cylindrical shell; $E=10^{7}, \nu=0.3$, $\rho=0.1, R=10, t=0.1, h=5$. 